\title{
The Extended Nambu-Jona-Lasinio Model and Hidden Local Symmetry of Low Energy QCD
}

\author{
M. Wakamatsu \\ Department of Physics, Faculty of Science, \\ Osaka University, \\ Toyonaka, Osaka 560, JAPAN \\ PACS numbers : 11.30.-j, 12.39.Fe, 12.40.Vv
}

\begin{abstract}
Using the standard auxiliary field method, we derive from the extended NambuJona-Lasinio model an effective meson action containing vector and axial-vector mesons in addition to Goldstone bosons. The vector and axial-vector mesons in this effective action transform as gauge fields of hidden local symmetry $G_{l o c a l}=\left[U(n)_{L} \times U(n)_{R}\right]_{l o c a l}$. Here, the realization of enlarged hidden local symmetry is accomplished via the introduction of two kinds of "compensating" fields. For obtaining the intrinsic-parity violating part of the action, we generalize the standard gauged Wess-Zumino-Witten action such that it also contains two kinds of "compensators" in addition to the usual Goldstone bosons as well as the vector and axial-vector mesons. This generalized gauged Wess-Zumino-Witten action turns out to have $G_{\text {globa }} \times G_{\text {local }}$ symmetry, where $G_{\text {global }}$ being the usual $U(n)_{L} \times U(n)_{R}$ global chiral symmetry
\end{abstract}


while $G_{\text {local }}$ being the $U(n)_{L} \times U(n)_{R}$ hidden local symmetry. This means that $G_{\text {local }}$ has no gauge anomaly and its associated vector and axial-vector mesons can be regarded as gauge bosons of $G_{\text {local }}$. The introduction of the coupling with the external electroweak fields requires us to gauge some appropriate subgroup of $G_{\text {global }}$. To perform it in consistent with the anomaly structure of QCD is a nontrivial problem. We explain how this can be done, following the recent suggestion by several authors.

\section{Introduction}

It is a widely-accepted belief that the applicable region of the nonlinear sigma model as a low energy effective theory of QCD can be extended to higher energies by incorporating other mesons (especially the vector and axial-vector mesons) than the Nambu-Goldstone bosons as explicit dynamical degrees of freedom. There are several ways to introduce spin-1 mesons into the basic chiral lagrangian [1-6]. Widely known examples includes the so-called massive Yang-Mills scheme as well as the scheme based on the hidden local symmetry initiated by Bando et al. [6-9]. Also known for a long time is a general theoretical framework based on the nonlinear realization of chiral symmetry initiated by Weinberg [10] and further developed by Callan, Coleman, Wess and Zumino [11]. Another approach, in which spin-1 mesons are represented as antisymmetric tensor fields, have also been proposed recently [12]. Although it is a general belief now that all of these approaches are in principle equivalent (see, for instance, the recent review by Birse [4]), the scheme based on the hidden gauge symmetry

has attracted special attention because of its several appealing features [5,6]. Bando et al's 
original construction of the model is based on the observation that a nonlinear sigma model based on the manifold $G / H=U(3)_{L} \times U(3)_{R} / U(n)_{V}$ is gauge equivalent to a "linear" model with $G_{\text {global }} \times H_{\text {local }}$ symmetry, where $H_{\text {local }}$ is the hidden local symmetry whose corresponding gauge fields are composite gauge bosons $[7,13,14]$. Later, Bando et al. enlarged the hidden local symmetry further into $G_{\text {local }}=U(3)_{L} \times U(3)_{R}$, which enables them to construct an effective lagrangian containing not only the vector mesons but also the axial-vector mesons [8,9]. A basic assumption in their construction is that the kinetic terms of such composite gauge bosons are generated through some quantum effects, as it actually happens for the $C P_{N-1}$ model [15]. Up to now, the validity of this scenario has been explicitly confirmed only within the extended Nambu-Jona-Lasinio (NJL) model as a tractable substitute of QCD lagrangian in the low energy domain [16-24]. In fact, the generation mechanism of the kinetic terms of spin-1 bosons has long been known in the auxiliary field treatment of the extended NJL lagrangian [25-27]. Some years ago, we have shown that an approximate bosonisation of the extended NJL lagrangian by using the auxiliary field method leads to a gauge fixed form of Bando et al's lagrangian with the enlarged hidden local symmetry $U(n)_{L} \times U(n)_{R}[21]$. A natural question is whether one can also obtain a corresponding lagrangian with explicit hidden gauge symmetry. It turns out that it is in fact possible if one introduces two kinds of compensating fields (or "compensators"), which plays the role of the gauge parameters to be absorbed into the masses of the vector and axial-vector mesons in the unitary gauge.

In this paper, we shall explicitly derive an effective meson lagrangian with the enlarged hidden local symmetry belonging to $U(n)_{L} \times U(n)_{R}$, by starting from the extended NJL model. Due to the presence of the $\gamma^{\mu} \gamma_{5}$ coupling between the quark fields and the auxiliary axialvector fields, we must pay special care to symmetries possessed by the original lagrangian, 
which may not be maintained in the resultant effective meson action due to the chiral anomaly. The original NJL lagrangian has global chiral symmetry. Since this is also a fundamental symmetry of strong interactions, we want to keep it unbroken after quark loop integral. On the other hand, the hidden local symmetry $G_{l o c a l}=\left[U(n)_{L} \times U(n)_{R}\right]_{l o c a l}$ is put into the formalism by introducing two kinds of compensating fields $[6,9]$. We nevertheless want to maintain this symmetry, since we can then obtain an effective gauge theory of vector and axial-vector mesons. The question is now whether it is possible to maintain both of these symmetries simultaneously. (That this is not a trivial question may be deduced from Bando et al's remark in [6] that in the case $G_{\text {local }}=\left[U(n)_{L} \times U(n)_{R}\right]_{\text {local }}$, the anomaly associated with $G_{\text {local }}$ should be canceled by an extra Wess-Zumino term, since QCD possesses the $G_{\text {global }}$ anomaly but not the $G_{\text {local }}$ anomaly at all.) As we shall see, if it were not for the couplings with the external electroweak gauge fields, an effective action with the desired symmetries can readily be obtained. However, once these couplings are introduced, a nontrivial problem arises, which has in fact caused much confusion in the past [28-31]. It seemed that within the framework of the extended NJL model there is no way to satisfy both conditions, i.e. the global chiral symmetry of hadronic processes and the electromagnetic gauge invariance [22,23]. Recently, a solution to this problem has been proposed by Bijnens and Prades [32]. (See also [33].) According to them, there is some uncertainty in the way the four quark vertex in the extended NJL model is treated. To be more explicit, they pointed out that the standardly assumed choice of the path integral measure corresponding to the hadronic vector and axial-vector fields are not necessarily justified. Their observation opens up a possibility to subtract local counter terms, which depend on both of the hadronic vector and axialvector fields and of the external electroweak gauge fields, to obtain an anomalous action with 
the desired symmetry. Making use of this observation, we can in fact obtain an anomalous action, which respects the global chiral symmetry at the strong interaction level as well as the electromagnetic gauge invariance, while keeping the full hidden local symmetry. To show that it is in fact possible is the main purpose of the present paper. We believe that this explicit construction will help us to deepen our understanding about the meaning of the hidden local symmetry in low energy effective theories of QCD.

The plan of the paper is as follows. In sect.2, we treat the case in which the electroweak couplings are switched off. The realistic case with the electroweak couplings will be discussed in sect 3. Sect.4 summarizes main results of the present study. Possible advantages in working in a theory with extra gauge degrees of freedom will also be discussed there.

\section{Extendend NJL model and its effective meson action}

\subsection{Definition of effective meson action}

Here we start with the following extended NJL model with its chirally invariant fourfermion couplings [16-24] :

$$
\begin{aligned}
\mathcal{L}_{N J L}=\bar{q} i \gamma^{\mu} \partial_{\mu} q & +2 G_{S} \sum_{a=1}^{n^{2}-1}\left\{\left(\bar{q} T^{a} q\right)^{2}+\left(\bar{q} i \gamma_{5} T^{a} q\right)^{2}\right\} \\
& -2 G_{V} \sum_{a=1}^{n^{2}-1}\left\{\left(\bar{q} \gamma^{\mu} T^{a} q\right)^{2}+\left(\bar{q} \gamma^{\mu} \gamma_{5} T^{a} q\right)^{2}\right\} .
\end{aligned}
$$

Here $q$ are the quark fields, $n$ is the number of the flavor degrees of freedom, and $T^{a}$ are generators of the flavor $U(n)$ group normalized as $\operatorname{tr}\left(T^{a} T^{b}\right)=\frac{1}{2} \delta_{a b}$. (The color indices of quarks are not shown explicitly.) Throughout the present study, we shall neglect the bare quark masses, for simplicity. In this chiral limit, the above lagrangian has exact chiral 
symmetry $\left[U(n)_{L} \times U(n)_{R}\right]_{\text {global }}$. (We also neglect the so-called $U_{A}(1)$ problem for reasons of simplicity [34].)

Introducing the color singlet collective (auxiliary) meson fields in the standard way, the lagrangian (2.1) can be rewritten as follows. First define $\mathcal{L}$ by

$$
\mathcal{L}=\mathcal{L}_{N J L}+\mathcal{L}_{\text {auxiliary }},
$$

with

$$
\begin{aligned}
\mathcal{L}_{\text {auxiliary }}= & -\frac{1}{8 G_{S}} \sum_{a}\left(S^{a}+4 G_{S} \bar{q} T^{a} q\right)^{2} \\
& -\frac{1}{8 G_{S}} \sum_{a}\left(P^{a}+4 G_{S} \bar{q} i \gamma_{5} T^{a} q\right)^{2} \\
& +\frac{1}{8 G_{V}} \sum_{a}\left(V^{a \mu}+4 G_{V} \bar{q} \gamma^{\mu} T^{a} q\right)^{2} \\
& +\frac{1}{8 G_{V}} \sum_{a}\left(A^{a \mu}+4 G_{V} \bar{q} \gamma^{\mu} \gamma_{5} T^{a} q\right)^{2} .
\end{aligned}
$$

Here $S^{a}, P^{a}, V^{a \mu}$, and $A^{a \mu}$ are collective scalar, pseudouscalar, vector and axial-vector fields. Defining the quantities (henceforth, the summation symbol for the repeated flavor index $a$ will be suppressed),

$$
S=S^{a} T^{a}, \quad P=P^{a} T^{a}, \quad V_{\mu}=-i V_{\mu}^{a} T^{a}, \quad A_{\mu}=-i A_{\mu}^{a} T^{a},
$$

we can write the lagrangian as

$$
\begin{aligned}
\mathcal{L}= & \bar{q}\left[i \gamma^{\mu}\left(\partial_{\mu}+V_{\mu}+\gamma_{5} A_{\mu}\right)-\left(S+i \gamma_{5} P\right)\right] q \\
& -\frac{1}{4 G_{S}} \operatorname{tr}\left[S^{2}+P^{2}\right]-\frac{1}{4 G_{V}} \operatorname{tr}\left[V_{\mu}^{2}+A_{\mu}^{2}\right] .
\end{aligned}
$$

Since there are no kinetic terms for the collective meson fields $S^{a}, P^{a}, V^{a \mu}$, and $A^{a \mu}$, they are auxiliary at this stage, and the lagrangian (2.5) is classically equivalent to the original one 
(2.1). It is now convenient to rewrite (2.5) using the left-right notation as $R_{\mu}=V_{\mu}+A_{\mu}$, $L_{\mu}=V_{\mu}-A_{\mu}$, and $M=S+i P$ :

$$
\begin{aligned}
\mathcal{L}= & \bar{q}\left[i \gamma^{\mu}\left(\partial_{\mu}+L_{\mu}+R_{\mu}\right)-\left(M^{\dagger} P_{L}+M P_{R}\right)\right] q \\
& -\frac{1}{4 G_{S}} \operatorname{tr} M M^{\dagger}-\frac{1}{8 G_{V}} \operatorname{tr}\left[L_{\mu}^{2}+R_{\mu}^{2}\right]
\end{aligned}
$$

where $P_{R / L}=\frac{1}{2}\left(1 \pm \gamma_{5}\right)$ being chirality projection operators. Customarily, the complex field $M$ is parameterized as

$$
M=\xi \Sigma \xi
$$

in terms of a hermitian matrix $\Sigma$ and a unitary matrix $\xi$, where the latter is written as $\xi(x)=e^{i \pi(x) / f_{\pi}}$ in terms of the Goldstone boson $\pi(x)=\pi^{a}(x) T^{a}$. According to Bando, Kugo, and Yamawaki [6], however, an arbitrary complex matrix can be expressed as a product of a unitary matrix $U$ and a positive hermitian matrix $\tilde{H}$, so that one may generally rewrite the complex matrix $M$ as

$$
M=U \tilde{H}=\xi_{L}^{\dagger} H \xi_{R}
$$

where the second equality is obtained by introducing the two unitary matrices $\xi_{L}$ and $\xi_{R}$ :

$$
\begin{aligned}
U & =\xi_{L}^{\dagger} \xi_{R}, \\
\tilde{H} & =\xi_{R}^{\dagger} H \xi_{R} .
\end{aligned}
$$

As pointed out by them, the decomposition of $U$ into $\xi_{L}$ and $\xi_{R}$ in (2.9) is not unique and this arbitrariness is related to the appearance of the hidden local symmetry $U(n)_{V}$ discussed below. The chiral transformation law of $M$ follows from the invariance of $\bar{\psi} M P_{R} \psi=\bar{\psi}_{L} M \psi_{R}$ :

$$
M \quad \longrightarrow \quad M^{\prime}=g_{L} M g_{R}^{\dagger}
$$


This , together with (2.8), (2.9), and (2.10), leads to the transformation laws of $\xi_{L, R}$ and $H$ :

$$
\begin{aligned}
& \xi_{L}(x) \longrightarrow \xi_{L}^{\prime}(x)=h(x) \xi_{L}(x) g_{L}^{\dagger} \\
& \xi_{R}(x) \longrightarrow \xi_{R}^{\prime}(x)=h(x) \xi_{R}(x) g_{R}^{\dagger} \\
& H(x) \longrightarrow H^{\prime}(x)=h(x) H(x) h^{\dagger}(x), \quad h(x) \in\left[U(n)_{V}\right]_{l o c a l}
\end{aligned}
$$

It is possible to further enlarge the above hidden local symmetry to $\left[U(n)_{L} \times U(n)_{R}\right]_{l o c a l}$ by introducing another dynamical variable $\xi_{M}(x)$ in such a way that $[8,9]$

$$
U(x)=\xi_{L}^{\dagger}(x) \xi_{M}(x) \xi_{R}(x)
$$

The transformation properties of $\xi_{L, R}$ and $\xi_{M}$ under $\left(g_{L}, g_{R}\right) \in\left[U(n)_{L} \times U(n)_{R}\right]_{g l o b a l}$ and $\left(h_{L}(x), h_{R}(x)\right) \in\left[U(n)_{L} \times U(n)_{R}\right]_{l o c a l}$ are given by

$$
\begin{aligned}
\xi_{L, R}(x) & \longrightarrow \xi_{L, R}^{\prime}(x)=h_{L, R}(x) \xi_{L, R} g_{L, R}^{\dagger} \\
\xi_{M}(x) & \longrightarrow \xi_{M}^{\prime}(x)=h_{L}(x) \xi_{M}(x) h_{R}^{\dagger}(x)
\end{aligned}
$$

The redundant nature of the representation (2.15) can most clearly be seen by introducing a parametrization as [9]

$$
\begin{aligned}
\xi_{L, R}(x) & =e^{ \pm i p(x) / f_{\pi}} \cdot e^{i \sigma(x) / f_{\pi}} \cdot e^{\mp i \pi(x) / f_{\pi}} \\
\xi_{M}(x) & =e^{2 i p(x) / f_{\pi}} .
\end{aligned}
$$

This reveals that the realization of the extended hidden local symmetry is accomplished via the introduction of two kinds of "compensating fields", $p(x)=p^{a}(x) T^{a}$ and $\sigma(x)=\sigma^{a}(x) T^{a}$, which play the role of the gauge parameters to be absorbed into the masses of the vector and axial-vector mesons in the unitary gauge such that

$$
p(x)=0 \quad \text { or } \quad \xi_{M}(x)=1,
$$


and further

$$
\sigma(x)=0 \quad \text { or } \quad \xi_{L}^{\dagger}(x)=\xi_{R}(x)=\xi(\pi)=e^{i \pi(x) / f_{\pi}}
$$

respectively.

From now on, we adopt the most general representation $(2.15)$ for $U(x)$, while replacing the hermitian matrix $H(x)$ by its vacuum expectation value $<H(x)>=m$ with $m$ being the dynamical quark mass generated through the spontaneous chiral symmetry breaking of the QCD vacuum. This latter approximation is motivated by the fact that we are interested in an effective meson action which does not contain physical scalar fields. (This is just the standard motivation for considering nonlinear realization of chiral symmetry [1-6].) The lagrangian (2.6) is then written as

$$
\begin{aligned}
\mathcal{L}= & \bar{q}\left[i \gamma^{\mu}\left(\partial_{\mu}+L_{\mu} P_{L}+R_{\mu} P_{L}\right)-m\left(\xi_{R}^{\dagger} \xi_{M}^{\dagger} \xi_{L} P_{L}+\xi_{L}^{\dagger} \xi_{M} \xi_{R} P_{R}\right)\right] q \\
& -\frac{1}{8 G_{V}} \operatorname{tr}\left[L_{\mu}^{2}+R_{\mu}^{2}\right]
\end{aligned}
$$

Here we have dropped an irrelevant constant term. It is convenient to introduce new fermion variables via a field dependent chiral rotation (Weinberg rotation) as

$$
\chi_{L}(x) \equiv \xi_{L}(x) q_{L}(x), \quad \chi_{R}(x) \equiv \xi_{R}(x) q_{R}(x)
$$

Then, we can rewrite the lagrangian as

$$
\begin{aligned}
\mathcal{L}= & \chi\left[i \gamma^{\mu}\left(\partial_{\mu}+\tilde{L}_{\mu} P_{L}+\tilde{R}_{\mu} P_{R}\right)-m\left(\xi_{M}^{\dagger} P_{L}+\xi_{M} P_{R}\right)\right] \chi \\
& -\frac{1}{8 G_{V}}\left[\operatorname{tr}\left(D_{L} \xi_{L} \cdot \xi_{L}^{\dagger}\right)^{2}+\operatorname{tr}\left(D_{R} \xi_{R} \cdot \xi_{R}^{\dagger}\right)^{2}\right]
\end{aligned}
$$

where

$$
\begin{aligned}
D_{L} \xi_{L} & =\left(\partial_{\mu}+\tilde{L}_{\mu}\right) \xi_{L}, \\
D_{R} \xi_{R} & =\underset{9}{\left(\partial_{\mu}+\tilde{R}_{\mu}\right) \xi_{R},}
\end{aligned}
$$


with the following definition of the new vector and axial-vector fields :

$$
\begin{aligned}
\tilde{L}_{\mu} & \equiv \xi_{L}\left(L_{\mu}+\partial_{\mu}\right) \xi_{L}^{\dagger}, \\
\tilde{R}_{\mu} & \equiv \xi_{R}\left(R_{\mu}+\partial_{\mu}\right) \xi_{R}^{\dagger} .
\end{aligned}
$$

The transformation laws of $\tilde{L}_{\mu}$ and $\tilde{R}_{\mu}$ follows from (2.27) and (2.28):

$$
\begin{aligned}
& \tilde{L}_{\mu} \longrightarrow \tilde{L}_{\mu}^{\prime}=h_{L}(x)\left(\tilde{L}_{\mu}+\partial_{\mu}\right) h_{L}^{\dagger}(x), \\
& \tilde{R}_{\mu} \longrightarrow \tilde{R}_{\mu}^{\prime}=h_{R}(x)\left(\tilde{R}_{\mu}+\partial_{\mu}\right) h_{R}^{\dagger}(x),
\end{aligned}
$$

which shows that they transform as gauge fields of the enlarged hidden local symmetry $\left[U(n)_{L} \times U(n)_{R}\right]^{(H L S)}$.

Two remarks are in order here by following Bando, Kugo, and Yamawaki [6]. First, at the stage of lagrangian (2.6), the vacuum functional $Z$ is given by

$$
Z=\int \mathcal{D} M \mathcal{D} M^{\dagger} \mathcal{D} L_{\mu} \mathcal{D} R_{\mu} \cdot Z_{f}
$$

with

$$
Z_{f}=\int \mathcal{D} q \mathcal{D} \bar{q} e^{i \int d^{4} x \mathcal{L}}
$$

When the variable $M$ is changed into $U$ and $\tilde{H}$ and further into $\xi_{L}, \xi_{R}, \xi_{M}$ and $H$, the path integral measure $\mathcal{D} M \mathcal{D} M^{\dagger}$ becomes

$$
\mathcal{D} M \mathcal{D} M^{\dagger}=\mathcal{D} U \mathcal{D} \tilde{H}=\mathcal{D} \xi_{L} \mathcal{D} \xi_{R} \mathcal{D} \xi_{M} \mathcal{D} H \delta\left(\xi_{L}^{\dagger}-\xi_{R}\right) \delta\left(\xi_{M}-1\right) .
$$

Here the delta function parts $\delta\left(\xi_{L}^{\dagger}-\xi_{R}\right)$ and $\delta\left(\xi_{M}-1\right)$ are necessary, since otherwise the number of degrees of freedom corresponding to the $U$ field would be tripled when expressing $U$ in terms of $\xi_{L}, \xi_{R}$ and $\xi_{M}$. This particular form of constraints corresponds to taking the unitary gauge (2.20) and (2.21). However, it is clear that the existence of the hidden local 
symmetry allows us to replace those constraints by more general gauge fixing conditions. Once this fact is understood, we can concentrate on the fermion part of the path integral $Z_{f}$, by leaving the gauge fixing problem for later consideration. Secondly, as is widely known, the fermion path integral measure $\mathcal{D} q \mathcal{D} \bar{q}$ is not invariant due to the presence of chiral anomaly [35]. Instead, we have

$$
\mathcal{D} q \mathcal{D} \bar{q}=\left(J_{1}\right)^{N_{c}} \mathcal{D} \chi \mathcal{D} \chi^{\dagger}
$$

where $\left(J_{1}\right)^{N_{c}}$ is the Jacobian of the transformation (2.23). If we carry out further change of fermion variables as

$$
\begin{aligned}
\varphi_{L}(x) & \equiv \xi_{M}^{\dagger}(x) \chi_{L}(x), \\
\varphi_{R}(x) & \equiv \quad \chi_{R}(x),
\end{aligned}
$$

then $Z_{f}$ can be written in the following three forms :

$$
\begin{aligned}
Z_{f} & =\int \mathcal{D} q \mathcal{D} \bar{q} e^{i \int d^{4} x \bar{q} D q} \\
& =J_{1}^{N_{c}} \int \mathcal{D} \chi \mathcal{D} \bar{\chi} e^{i \int d^{4} x \bar{\chi} \tilde{D} \chi} \\
& =J_{1}{ }^{N_{c}} J_{2}{ }^{N_{c}} \int \mathcal{D} \varphi \mathcal{D} \bar{\varphi} e^{i \int d^{4} x \bar{\varphi} \hat{D} \varphi},
\end{aligned}
$$

with

$$
\begin{aligned}
& D=i \gamma^{\mu}\left(\partial_{\mu}+L_{\mu} P_{L}+R_{\mu} P_{R}\right)-m\left(\xi_{R}^{\dagger} \xi_{M}^{\dagger} \xi_{L} P_{L}+\xi_{L}^{\dagger} \xi_{M} \xi_{R} P_{R}\right), \\
& \tilde{D}=i \gamma^{\mu}\left(\partial_{\mu}+\tilde{L}_{\mu} P_{L}+\tilde{R}_{\mu} P_{R}\right)-m\left(\xi_{M}^{\dagger} P_{L}+\xi_{M} P_{R}\right), \\
& \hat{D}=i \gamma^{\mu}\left(\partial_{\mu}+\hat{L}_{\mu} P_{L}+\hat{R}_{\mu} P_{R}\right)-m .
\end{aligned}
$$

Here we have defined the new field variables as

$$
\begin{aligned}
& \hat{L}_{\mu} \equiv \xi_{M}^{\dagger}\left(\tilde{L}_{\mu}+\partial_{\mu}\right) \xi_{M}, \\
& \hat{R}_{\mu} \equiv \tilde{R}_{\mu},
\end{aligned}
$$


whereas $J_{2}{ }^{N_{c}}$ in (2.45) is the Jacobian of the transformation (2.35) and (2.36). Formally carrying out the fermion path integral, we then obtain

$$
\begin{aligned}
Z_{f} & =(\operatorname{det} D)^{N_{c}} \\
& =\left(J_{1}\right)^{N_{c}}(\operatorname{det} \tilde{D})^{N_{c}} \\
& =\left(J_{1}\right)^{N_{c}}\left(J_{2}\right)^{N_{c}}(\operatorname{det} \hat{D})^{N_{c}} .
\end{aligned}
$$

As is well known, the real part of $\log (\operatorname{det} D)^{N_{c}}$ contributes to the non-anomalous (intrinsicparity conserving) part of effective action, whereas the imaginary part of it gives the anomalous (intrinsic-parity violating) part $[18,19]$. (Here and hereafter, we frequently use the terminology as above in the Euclidean formulation of the path integral for convenience, in spite that we are working in the Minkowski formulation.) We also know that the modulus of the quark determinant is chiral gauge invariant $[18,19]$, i.e.

$$
|\operatorname{det} D|=|\operatorname{det} \tilde{D}|=|\operatorname{det} \hat{D}| \text {. }
$$

This part contains divergences, which must be removed by some regularization procedure. Here we adopt the proper-time regularization scheme with some intrinsic cutoff $\Lambda$ :

$$
\begin{aligned}
N_{c} \log |\operatorname{det} D| & =\frac{N_{c}}{2} \operatorname{Tr}^{\prime} \log D^{\dagger} D \\
& \longrightarrow-\frac{N_{c}}{2} \int_{1 / \Lambda^{2}}^{\infty} \frac{d \tau}{\tau} \operatorname{Tr}^{\prime} e^{-\tau D^{\dagger} D}
\end{aligned}
$$

Here $\operatorname{Tr}=\int d^{4} x \operatorname{tr}$ and a prime on it indicates that a trace over Dirac indices is included. $D^{\dagger} D$ in the above equation can naturally be replaced by either of $\tilde{D}^{\dagger} \tilde{D}$ or $\hat{D}^{\dagger} \hat{D}$. It is also clear from (2.40) that det $\hat{D}$ has no imaginary part or it is chiral gauge invariant, so that the imaginary part $\Delta$ of $\log \operatorname{det} D$ comes from the two Jacobians $J_{1}$ and $J_{2}$, leading to the 
following expression :

$$
\log (\operatorname{det} D)^{N_{c}}=N_{c} \log |\operatorname{det} D|+i \Delta
$$

with

$$
\Delta=N_{c} \operatorname{Im}\left(\log J_{1}+\log J_{2}\right) .
$$

The real and imaginary parts of the effective meson action will be discussed separately in the following two subsections.

\subsection{Non-anomalous effective action}

Since our main concern in this paper is the anomalous part of the action, we give here only a brief survey of the nonanomalous part of it, which can be obtained by using the standard derivative expansion method [18-21]. As already pointed out, it is immaterial which form of Dirac operator, i.e. the original one $D$, or the chirally rotated ones $\tilde{D}$ or $\hat{D}$, is used in this evaluation (at least assuming infinite summation of the gradient expansion). It is a matter

of representation. Here we adopt the form $\tilde{D}$, since we want to interpret $\tilde{L}_{\mu}$ and $\tilde{R}_{\mu}$ as gauge bosons of the hidden local $U(n)_{L} \times U(n)_{R}$ symmetry. (Remember the transformation laws (2.29) and (2.30) of $\tilde{\mathcal{L}}_{\mu}$ and $\tilde{\mathcal{R}}_{\mu}$.) As follows is the outline of the necessary manipulation $[20,21]$. First, truncate the derivative expansion at terms of second order. Second, introduce the coupling constant $g_{V}$ by the relation

$$
g_{V}=\left\{\frac{2}{3} \frac{N_{c}}{(4 \pi)^{2}} \Gamma\left(0, \frac{m^{2}}{\Lambda^{2}}\right)\right\}^{-1 / 2},
$$

where $\Gamma(\alpha, x)$ is the incomplete gamma function defined by $\Gamma(\alpha, x)=\int_{x}^{\infty} d t e^{-t} t^{\alpha-1}$. Then, examining the coefficients of the bilinear terms of the field variables in the resultant effective 
lagrangian, we find the following relations :

$$
M_{V}^{2}=\frac{g_{V}^{2}}{4 G_{V}}, \quad M_{A}^{2}=M_{V}^{2}+6 m^{2}, \quad f_{\pi}^{2}=\frac{1}{4 G_{V}}\left(1-\frac{M_{V}^{2}}{M_{A}^{2}}\right) .
$$

Here $M_{V}$ and $M_{A}$ are respectively the masses of the vector and axial-vector mesons, with $m$ being the dynamical quark mass [16-22]. Finally, defining the parameter $a$ by the equation $[20,21]$

$$
a=\left(1-\frac{M_{V}^{2}}{M_{A}^{2}}\right)^{-1},
$$

we are led to an effective meson lagrangian of the following form :

$$
\Gamma^{(n)}=\int d^{4} x \mathcal{L}^{(n)}
$$

with

$$
\begin{aligned}
\mathcal{L}^{(n)}= & \frac{1}{4 g_{V}^{2}} \operatorname{tr}\left[\tilde{L}_{\mu \nu}^{2}+\tilde{R}_{\mu \nu}^{2}\right]-\frac{a}{a-1} \frac{f_{\pi}^{2}}{4} \operatorname{tr}\left[D_{\mu} \xi_{M} \cdot \xi_{M}^{\dagger}\right]^{2} \\
& -\frac{1}{2} a f_{\pi}^{2} \operatorname{tr}\left[D_{\mu} \xi_{L} \cdot \xi_{L}^{\dagger}\right]^{2}-\frac{1}{2} a f_{\pi}^{2} \operatorname{tr}\left[D_{\mu} \xi_{R} \cdot \xi_{R}^{\dagger}\right]^{2} .
\end{aligned}
$$

Here

$$
\begin{aligned}
& \tilde{L}_{\mu \nu}=\partial_{\mu} \tilde{L}_{\nu}-\partial_{\nu} \tilde{L}_{\mu}+\left[\tilde{L}_{\mu}, \tilde{L}_{\nu}\right], \\
& \tilde{R}_{\mu \nu}=\partial_{\mu} \tilde{R}_{\nu}-\partial_{\nu} \tilde{R}_{\mu}+\left[\tilde{R}_{\mu}, \tilde{R}_{\nu}\right],
\end{aligned}
$$

and

$$
\begin{aligned}
D_{\mu} \xi_{L} & =\partial_{\mu} \xi_{L}+\tilde{L}_{\mu} \xi_{L}, \\
D_{\mu} \xi_{R} & =\partial_{\mu} \xi_{R}+\tilde{R}_{\mu} \xi_{R}, \\
D_{\mu} \xi_{M} & =\partial_{\mu} \xi_{M}+\tilde{L}_{\mu} \xi_{M}-\xi_{M} \tilde{R}_{\mu} .
\end{aligned}
$$


The lagrangian above may be compared with that of Bando et al. derived from the symmetry principle of enlarged hidden local symmetry [9] :

$$
\mathcal{L}=\frac{1}{4 g_{V}^{2}} \operatorname{tr}\left[\tilde{L}_{\mu \nu}^{2}+\tilde{R}_{\mu \nu}^{2}\right]+a^{\prime} \mathcal{L}_{V}+b^{\prime} \mathcal{L}_{A}+c^{\prime} L_{M}+d^{\prime} \mathcal{L}_{\pi}
$$

where

$$
\begin{aligned}
\mathcal{L}_{V} & =-\frac{f_{\pi}^{2}}{4} \operatorname{tr}\left[D_{\mu} \xi_{L} \cdot \xi_{L}^{\dagger}+\xi_{M} D_{\mu} \xi_{R} \cdot \xi_{R}^{\dagger} \xi_{M}^{\dagger}\right]^{2} \\
\mathcal{L}_{A} & =-\frac{f_{\pi}^{2}}{4} \operatorname{tr}\left[D_{\mu} \xi_{L} \cdot \xi_{L}^{\dagger}-\xi_{M} D_{\mu} \xi_{R} \cdot \xi_{R}^{\dagger} \xi_{M}^{\dagger}\right]^{2} \\
\mathcal{L}_{M} & =-\frac{f_{\pi}^{2}}{4} \operatorname{tr}\left[D_{\mu} \xi_{M} \cdot \xi_{M}^{\dagger}\right]^{2}, \\
\mathcal{L}_{\pi} & =-\frac{f_{\pi}^{2}}{4} \operatorname{tr}\left[D_{\mu} \xi_{L} \cdot \xi_{L}^{\dagger}-\xi_{M} D_{\mu} \xi_{R} \cdot \xi_{R}^{\dagger} \xi_{M}^{\dagger}-D_{\mu} \xi_{M} \cdot \xi_{M}^{\dagger}\right]^{2} .
\end{aligned}
$$

It is easy to see that their lagrangian (2.60) coincides with our lagrangian (2.54), apart from the fact that the arbitrary constants $a^{\prime}, b^{\prime}, c^{\prime}, d^{\prime}$ of theirs are respectively constrained such that $a^{\prime}=b^{\prime}=a, c^{\prime}=a /(a-1), d^{\prime}=0$ in our effective lagrangian, which has been derived from a specific underlying lagrangian at the quark level. Note that, from our derivation here, it is self-evident that the equality of $a^{\prime}$ and $b^{\prime}$ is simply a reflection of the chiral symmetry satisfied by the original NJL lagrangian [21].

\subsection{Anomalous effective action}

Now we turn to the discussion of the intrinsic-parity violating part of the effective action, which is of our primary concern in this paper :

$$
\begin{aligned}
\Gamma^{(a)} & =N_{c} \operatorname{Im} \log \operatorname{det} D \\
& =-i N_{c}\left(\log J_{1}+\log J_{2}\right) .
\end{aligned}
$$


Here use has been made of the fact that the Jacobians $J_{1}$ and $J_{2}$ are pure phase $[18,19]$. It is easier to first evaluate the response of the Jacobians under small chiral variations of the relevant fields, rather than calculating the Jacobians directly. The answer depends on the regularization scheme. Since the presence of anomaly does not allow us to maintain all the symmetries of the classical level lagrangian, a general question is what symmetries should be kept unbroken after regularization. At the classical level, our lagrangian has the $U(n)_{L} \times U(n)_{R}$ global symmetry and the $U(n)_{L} \times U(n)_{R}$ hidden local symmetries. One might then wonder which symmetry should be kept unbroken in our particular problem. Fortunately, in the absence of the coupling with the external electroweak fields, the answer is quite simple. In fact, we can retain both of these symmetries despite the presence of the anomaly. (As a matter of course, what is truly interesting from the physical viewpoint is the realistic case with the electroweak couplings. We discuss this physically interesting problem separately in the next section.)

Let us now construct the anomalous action explicitly, which has $\left[U(n)_{L} \times U(n)_{R}\right]_{g l o b a l}^{(e x t)}$ and $\left[U(n)_{L} \times U(n)_{R}\right]^{(H L S)}$ symmetries. The most elegant way to carry out this program is to use the differential geometric method [36-39]. (Here we closely follow the treatment by Petersen [39].) For convenience sake, let us consider space-time dependent chiral transformations $\left(g_{L}(x), g_{R}(x)\right) \in\left[U(n)_{L} \times U(n)_{R}\right]_{\text {local }}^{(e x t)}$, which generalizes the global chiral transformation. Its infinitesimal form is given as

$$
\begin{aligned}
& g_{L}(x)=e^{-\theta_{L}(x)} \simeq 1-\theta_{L}(x) \\
& g_{R}(x)=e^{-\theta_{R}(x)} \simeq 1-\theta_{R}(x)
\end{aligned}
$$

with the property $\theta_{L}^{\dagger}=-\theta_{L}, \theta_{R}^{\dagger}=-\theta_{R}$. We list below the transformation properties of the 
relevant fields under this transformation :

$$
\begin{array}{ll}
\xi_{L}(x) \longrightarrow \xi_{L}(x) g_{L}^{\dagger}(x), & \text { or } \delta^{(e x t)} \xi_{L}=\xi_{L} \theta_{L}, \\
\xi_{R}(x) \longrightarrow \xi_{R}(x) g_{R}^{\dagger}(x), & \text { or } \delta^{(e x t)} \xi_{R}=\xi_{R} \theta_{R}, \\
\xi_{M}(x) \longrightarrow \xi_{M}(x), & \text { or } \delta^{(e x t)} \xi_{M}=0, \\
L(x) \longrightarrow g_{L}(x)(L(x)+d) g_{L}^{\dagger}(x), & \text { or } \delta^{(e x t)} L=d \theta_{L}+\left[L, \theta_{L}\right], \\
R(x) \longrightarrow g_{R}(x)(R(x)+d) g_{R}^{\dagger}(x), & \text { or } \delta^{(e x t)} R=d \theta_{R}+\left[R, \theta_{R}\right], \\
\tilde{L}(x) \longrightarrow \tilde{L}(x), & \text { or } \delta^{(e x t)} \tilde{L}=0, \\
\tilde{R}(x) \longrightarrow \tilde{R}(x), & \text { or } \delta^{(e x t)} \tilde{R}=0, \\
\hat{L}(x) \longrightarrow \hat{L}(x), & \text { or } \delta^{(e x t)} \hat{L}=0, \\
\hat{R}(x) \longrightarrow \hat{R}(x), & \text { or } \delta^{(e x t)} \hat{R}=0 .
\end{array}
$$

Here and hereafter, we use the notation of differential form with the definition $L=L_{\mu} d x^{\mu}$, $R=R_{\mu} d x^{\mu}$ and $d=d x^{\mu} \partial_{\mu}$ etc.

Similarly, under the transformation $\left(h_{L}(x), h_{R}(x)\right) \in\left[U(n)_{L} \times U(n)_{R}\right]^{(H L S)}$ with

$$
\begin{aligned}
& h_{L}(x)=e^{-\epsilon_{L}(x)} \simeq 1-\epsilon_{L}(x), \\
& h_{R}(x)=e^{-\epsilon_{R}(x)} \simeq 1-\epsilon_{R}(x),
\end{aligned}
$$

the relevant fields transform as

$$
\begin{array}{ll}
\xi_{L}(x) \longrightarrow h_{L}(x) \xi_{L}(x), & \text { or } \delta^{(H L S)} \xi_{L}=-\epsilon_{L} \xi_{L}, \\
\xi_{R}(x) \longrightarrow h_{R}(x) \xi_{R}(x), & \text { or } \delta^{(H L S)} \xi_{R}=-\epsilon_{R} \xi_{R}, \\
\xi_{M}(x) \longrightarrow h_{L}(x) \xi_{M}(x) h_{R}^{\dagger}(x), & \text { or } \delta^{(H L S)} \xi_{M}=\xi_{M} \epsilon_{R}-\epsilon_{L} \xi_{M}, \\
L(x) \longrightarrow L(x), & \text { or } \delta^{(H L S)} L(x)=0,
\end{array}
$$




$$
\begin{aligned}
& R(x) \longrightarrow R(x), \quad \text { or } \quad \delta^{(H L S)} R(x)=0, \\
& \tilde{L}(x) \longrightarrow h_{L}(x)(\tilde{L}(x)+d) h_{L}^{\dagger}(x), \quad \text { or } \quad \delta^{(H L S)} \tilde{L}=d \epsilon_{L}+\left[\tilde{L}, \epsilon_{L}\right], \\
& \tilde{R}(x) \longrightarrow h_{R}(x)(\tilde{R}(x)+d) h_{R}^{\dagger}(x), \quad \text { or } \quad \delta^{(H L S)} \tilde{R}=d \epsilon_{R}+\left[\tilde{R}, \epsilon_{R}\right], \\
& \hat{L}(x) \longrightarrow h_{R}(x)(\hat{L}(x)+d) h_{R}^{\dagger}(x), \quad \text { or } \quad \delta^{(H L S)} \hat{L}=d \epsilon_{R}+\left[\hat{L}, \epsilon_{R}\right], \\
& \hat{R}(x) \longrightarrow h_{R}(x)(\hat{R}(x)+d) h_{R}^{\dagger}(x), \quad \text { or } \quad \delta^{(H L S)} \hat{R}=d \epsilon_{R}+\left[\hat{R}, \epsilon_{R}\right] .
\end{aligned}
$$

The basic differential geometric object, which plays important roles in the following construction is the so-called Chern-Simons secondary form defined as

$$
\omega_{2 n+1}^{0}\left(A_{L}, A_{R}\right)=(n+1) \int_{-1}^{1} d t \operatorname{tr}\left[\dot{A}(t) F^{n}(t)\right],
$$

where $\dot{A}(t)=(d / d t) A(t)$ with

$$
\begin{aligned}
& A(t)=\frac{1}{2}\left(A_{L}+A_{R}\right)-\frac{1}{2}\left(A_{L}-A_{R}\right) t, \\
& F(t)=d A(t)+A^{2}(t),
\end{aligned}
$$

and $n=D / 2$ with $D$ being the space-time dimension. (We are of course interested in the case with $D=4$.) The special choice of the above integral path in the field space (the straight line connecting $A_{L}$ and $\left.A_{R}\right)$ dictates that $\omega_{5}^{0}\left(A_{L}, A_{R}\right)$ is invariant under the vector-type gauge transformation of $A_{L}$ and $A_{R}$, or that it gives the so-called Bardeen anomaly. We also need the following quantity defined by the above $\omega_{5}^{0}\left(A_{L}, A_{R}\right)$ as

$$
\hat{\omega}_{5}^{0}\left(A_{L}, A_{R}\right) \equiv \omega_{5}^{0}\left(A_{L}, 0\right)-\omega_{5}^{0}\left(A_{R}, 0\right) .
$$

This quantity is not invariant under either of the vector-type or axial-vector-type gauge transformation, whereas it gives the so-called left-right symmetric form of anomaly. An important observation is that the difference of $\omega_{5}^{0}\left(A_{L}, A_{R}\right)$ and $\hat{\omega}_{5}^{0}\left(A_{L}, A_{R}\right)$ is an exact form. 
In fact, it is known that one can write as [39]

$$
\omega_{5}^{0}\left(A_{L}, A_{R}\right)-\hat{\omega}_{5}^{0}\left(A_{L}, A_{R}\right)=d \rho_{4}\left(0, A_{L}, A_{R}\right)
$$

where

$$
\rho_{2 n}\left(A_{0}, A_{1}, A_{2}\right)=-(n+1) \sum_{p=0}^{n-1} \int_{0}^{1} d s \int_{0}^{1-s} d t \operatorname{tr}\left\{A_{2} F^{p}(s, t) A_{1} F^{n-p-1}(s, t)\right\}
$$

with

$$
\begin{aligned}
& A(s, t)=A_{0}+s A_{1}+t A_{2}, \\
& F(s, t)=d A(s, t)+A^{2}(s, t) .
\end{aligned}
$$

Specializing to the case of $D=4$ with $A_{0}=0, A_{1}=A_{L}, A_{2}=A_{R}$, we obtain

$$
\begin{aligned}
\rho_{4}\left(0, A_{L}, A_{R}\right)= & \frac{1}{2} \operatorname{tr}\left[\left(A_{L} A_{R}-A_{R} A_{L}\right)\left(F_{L}+F_{R}\right)\right. \\
& \left.-A_{L}^{3} A_{R}+A_{R}^{3} A_{L}+\frac{1}{2} A_{L} A_{R} A_{L} A_{R}\right] .
\end{aligned}
$$

(This is what we need later for writing down the explicit form of anomalous action with the required symmetry.) Using these differential geometric objects, the anomalous part of the action, which satisfies the requirements above, i.e. the invariance under $\left[U(n)_{L} \times U(n)_{R}\right]_{g l o b a l}^{(e x t)}$ and $\left[U(n)_{L} \times U(n)_{R}\right]^{(H L S)}$, can easily be written down as

$$
\begin{aligned}
\Gamma^{(a)} & =-i N_{c}\left(\log J_{1}+\log J_{2}\right) \\
& =c^{\prime} \int_{B^{5}}\left\{\left[\hat{\omega}_{5}^{0}(L, R)-\omega_{5}^{0}(\tilde{L}, \tilde{R})\right]+\left[\omega_{5}^{0}(\tilde{L}, \tilde{R})-\omega_{5}^{0}(\hat{L}, \hat{R})\right]\right\} \\
& =c^{\prime} \int_{B^{5}}\left[\hat{\omega}_{5}^{0}(L, R)-\omega_{5}^{0}(\hat{L}, \hat{R})\right],
\end{aligned}
$$

where $c^{\prime}=i\left(N_{c} / 24 \pi^{2}\right)$, while $B^{5}$ is a five-dimensional manifold with the four dimensional space-time as its boundary. Here $\left[\hat{\omega}_{5}^{0}(L, R)-\omega_{5}^{0}(\tilde{L}, \tilde{R})\right]$ part corresponds to the contribution from the Jacobian $J_{1}$, while $\left[\omega_{5}^{0}(\tilde{L}, \tilde{R})-\omega_{5}^{0}(\hat{L}, \hat{R})\right]$ from $J_{2}$. 
The fact that the above $\Gamma^{(a)}$ has the required properties can be convinced as follows. First notice that, under the $\left[U(n)_{L} \times U(n)_{R}\right]_{\text {local }}^{(e x t)}$ transformation, $L$ and $R$ transform according to (2.71) and (2.72), whereas $\hat{L}$ and $\hat{R}$ are absolutely intact under the same transformation. One then sees that only the $\omega_{5}^{0}(L, R)$ part in the last equation of $(2.89)$ changes under this transformation, which just gives the left-right symmetric form of anomaly by construction. This means that $\Gamma_{(a)}$ is invariant under the global chiral transformation, which is a special case of $\left[U(n)_{L} \times U(n)_{R}\right]_{\text {local }}^{(e x t)}$.

On the other hand, under the $\left[U(n)_{L} \times U(n)_{R}\right]^{(H L S)}$ transformation, $L$ and $R$ do not change, while $\hat{L}$ and $\hat{R}$ transform according to (2.86) and (2.87). Here, a crucial observation is that $\hat{L}$ and $\hat{R}$ transform exactly in the same manner. In other words, for the fields $\hat{L}$ and $\hat{R}$ introduced by (2.41) and (2.42), only the vector-type transformation is induced by arbitrary $\left[U(n)_{L} \times U(n)_{R}\right]^{(H L S)}$ transformation . Since $\omega_{5}^{0}(\hat{L}, \hat{R})$ is vector gauge invariant by construction, one then concludes that $\Gamma^{(a)}$ is invariant under $\left[U(n)_{L} \times U(n)_{R}\right]^{(H L S)}$, which insists that there is no anomaly in the enlarged hidden local symmetry.

Although the promised effective action has already been given in a formal sense, it is desirable to write down its explicit form. This is especially so, because our goal is to express the effective action in terms of $\xi_{L}, \xi_{R}, \xi_{M}, \tilde{L}$ and $\tilde{R}$, which are the dynamical variables of the enlarged hidden local symmetry scheme (or representation). It can be achieved by considering the integral path in the field space as illustrated in fig.1(a). This integral path is obtained by combining the two paths shown in fig.2(b), which are respectively related to the contribution from the Jacobians $J_{1}$ and $J_{2}$. From fig.1(a), we obtain

$$
\omega_{5}^{0}(L, 0)+\omega_{5}^{0}(0, R)+\omega_{5}^{0}\left(R, \xi_{R}^{\dagger} d \xi_{R}\right)+\omega_{5}^{0}\left(\xi_{R}^{\dagger} d \xi_{R}, 0\right)
$$




$$
\begin{aligned}
& +\omega_{5}^{0}(0, \tilde{R})+\omega_{5}^{0}(\hat{R}, \hat{L})+\omega_{5}^{0}(\hat{L}, 0)+\omega_{5}^{0}\left(0, \xi_{M} d \xi_{M}^{\dagger}\right) \\
& +\omega_{5}^{0}\left(\xi_{M} d \xi_{M}^{\dagger}, \tilde{L}\right)+\omega_{5}^{0}(\tilde{L}, 0)+\omega_{5}^{0}\left(0, \xi_{L}^{\dagger} d \xi_{L}\right)+\omega_{5}^{0}\left(\xi_{L}^{\dagger} d \xi_{L}, L\right) \\
& =d\left\{\rho_{4}\left(0, R, \xi_{R}^{\dagger} d \xi_{R}\right)+\rho_{4}(0, \hat{R}, \hat{L})+\rho_{4}\left(0, \xi_{M} d \xi_{M}^{\dagger}, \tilde{L}\right)+\rho_{4}\left(0, \xi_{L}^{\dagger} d \xi_{L}, L\right)\right\} .
\end{aligned}
$$

Now we can make use of the vector gauge invariant property of $\omega_{5}^{0}\left(A_{L}, A_{R}\right)$. For instance, we can show that

$$
\begin{aligned}
\omega_{5}^{0}\left(R, \xi_{R}^{\dagger} d \xi_{R}\right) & =T\left(\xi_{R}, \xi_{R}\right) \omega_{5}^{0}\left(R, \xi_{R}^{\dagger} d \xi_{R}\right) \\
& =\omega_{5}^{0}\left(\xi_{R}(R+d) \xi_{R}^{\dagger}, 0\right)=\omega_{5}^{0}(\tilde{R}, 0),
\end{aligned}
$$

where $T\left(g_{L}, g_{R}\right)$ defines the action of the element $g=g_{L} P_{L}+g_{R} P_{L} \in U(n)_{L} \times U(n)_{R}$. Then, by utilizing the antisymmetry of $\omega_{5}^{0}\left(A_{L}, A_{R}\right)$ with respect to the interchange of $A_{L}$ and $A_{R}$, i.e. $\omega_{5}^{0}\left(A_{L}, A_{R}\right)=-\omega_{5}^{0}\left(A_{R}, A_{L}\right)$, we find

$$
\omega_{5}^{0}\left(R, \xi_{R}^{\dagger} d \xi_{R}\right)+\omega_{5}^{0}(0, \tilde{R})=0 .
$$

Similarly, it is easy to verify that

$$
\begin{aligned}
& \omega_{5}^{0}(\hat{L}, 0)+\omega_{5}^{0}\left(\xi_{M} d \xi_{M}^{\dagger}, \tilde{L}\right)=0, \\
& \omega_{5}^{0}(\tilde{L}, 0)+\omega_{5}^{0}\left(\xi_{L}^{\dagger} d \xi_{L}, L\right)=0 .
\end{aligned}
$$

Using these equalities, we obtain from (2.98)

$$
\begin{aligned}
& \hat{\omega}_{5}^{0}(L, R)-\omega_{5}^{0}(\hat{L}, \hat{R}) \\
= & \omega_{5}^{0}\left(0, \xi_{R}^{\dagger} d \xi_{R}\right)-\omega_{5}^{0}\left(0, \xi_{L}^{\dagger} d \xi_{L}\right)-\omega_{5}^{0}\left(0, \xi_{M} d \xi_{M}^{\dagger}\right) \\
+ & d\left\{\rho_{4}\left(0, R, \xi_{R}^{\dagger} d \xi_{R}\right)+\rho_{4}\left(0, \xi_{L}^{\dagger} d \xi_{L}, L\right)+\rho_{4}\left(0, \xi_{M} d \xi_{M}^{\dagger}, \tilde{L}\right)-\rho_{4}(0, \hat{L}, \hat{R})\right\}
\end{aligned}
$$

where use has been made of the relation (2.91). Upon integration over the 5-dimensional 
manifold with space-time boundary, we then arrive at

$$
\begin{aligned}
\Gamma^{(a)} \equiv c^{\prime} \int_{B^{5}}\left[\hat{\omega}_{5}^{0}(L, R)-\omega_{5}^{0}(\hat{L}, \hat{R})\right] \\
=c^{\prime} \int_{B^{5}}\left[\omega_{5}^{0}\left(0, \xi_{R}^{\dagger} d \xi_{R}\right)-\omega_{5}^{0}\left(0, \xi_{L}^{\dagger} d \xi_{L}\right)-\omega_{5}^{0}\left(0, \xi_{M} d \xi_{M}^{\dagger}\right)\right] \\
+c^{\prime} \int_{S^{4}}\left[\rho_{4}\left(0, R, \xi_{R}^{\dagger} d \xi_{R}\right)+\rho_{4}\left(0, \xi_{L}^{\dagger} d \xi_{L}, L\right)\right. \\
\left.\quad+\rho_{4}\left(0, \xi_{M} d \xi_{M}^{\dagger}, \tilde{L}\right)-\rho_{4}(0, \hat{L}, \hat{R})\right]
\end{aligned}
$$

or equivalently

$$
\begin{gathered}
\Gamma^{(a)}=c^{\prime} \int_{B^{5}}\left[\omega_{5}^{0}\left(0, \xi_{R}^{\dagger} d \xi_{R}\right)-\omega_{5}^{0}\left(0, \xi_{L}^{\dagger} d \xi_{L}\right)-\omega_{5}^{0}\left(0, \xi_{M} d \xi_{M}^{\dagger}\right)\right] \\
+c^{\prime} \int_{S^{4}}\left[\rho_{4}\left(0, \xi_{R}^{\dagger}(\tilde{R}+d) \xi_{R}, \xi_{R}^{\dagger} d \xi_{R}\right)+\rho_{4}\left(0, \xi_{L}^{\dagger} d \xi_{L}, \xi_{L}^{\dagger}(\tilde{L}+d) \xi_{L}\right)\right. \\
\left.+\rho_{4}\left(0, \xi_{M} d \xi_{M}^{\dagger}, \tilde{L}\right)-\rho_{4}\left(0, \xi_{M}^{\dagger}(\tilde{L}+d) \xi_{M}, \tilde{R}\right)\right] .
\end{gathered}
$$

This is a desired effective action, which is expressed in terms of the dynamical variables $\xi_{L}$, $\xi_{R}, \xi_{M}, \tilde{L}$ and $\tilde{R}$ of the enlarged hidden local symmetry scheme. (We recall the explicit form of $\rho_{4}\left(0, A_{L}, A_{R}\right)$ given in (2.96).) The symmetries of the above action are characterized by its responses to arbitrary gauge variations belonging to $\left[U(n)_{L} \times U(n)\right]^{(e x t)}$ as well as $\left[U(n)_{L} \times U(n)_{R}\right]^{(H L S)}$. Under the independent left $\left(\theta_{L} \neq 0, \theta_{R}=0\right)$ and $\operatorname{right}\left(\theta_{L}=0, \theta_{R} \neq 0\right)$ gauge variations belonging to $\left[U(n)_{L} \times U(n)_{R}\right]^{(e x t)}$, we respectively obtain

$$
\begin{aligned}
& \delta_{L}^{(e x t)} \Gamma^{(a)}=c^{\prime} \int_{S^{4}} \operatorname{tr} d \theta_{L} \frac{1}{2}\left(d L L+L d L+L^{3}\right), \\
& \delta_{R}^{(e x t)} \Gamma^{(a)}=-c^{\prime} \int_{S^{4}} \operatorname{tr} d \theta_{R} \frac{1}{2}\left(d R R+R d R+R^{3}\right) .
\end{aligned}
$$

This just corresponds to the familiar left-right symmetric form of anomaly [36-39]. Specializing to global chiral transformations, which dictates that $d \theta_{L}=d \theta_{R}=0$, the above variations identically vanish. This means that $\Gamma^{(a)}$ maintains global chiral symmetry. On the other 
hand, one can verify that, under the transformation belonging to $\left[U(n)_{L} \times U(n)_{R}\right]^{(H L S)}, \Gamma^{(a)}$ is completely invariant, i.e.

$$
\delta_{L}^{(H L S)} \Gamma^{(a)}=\delta_{R}^{(H L S)} \Gamma^{(a)}=0
$$

Although these symmetry properties are obvious from the construction explained above, an explicit proof is given in Appendix A, for completeness, by calculating the gauge variation of each term of $\Gamma^{(a)}$. At any rate, the effective action (2.104) or (2.105) has complete gauge invariance under $\left[U(n)_{L} \times U(n)_{R}\right]^{(H L S)}$, so that one can work in any gauge one wants. Here, an especially interesting gauge is such that $\xi_{L}=\xi_{M}=1$, and $\xi_{R}=U$. In this special gauge, the action $(2.105)$ reduces to

$$
\begin{aligned}
\Gamma^{(a)} \longrightarrow & c^{\prime} \int_{B^{5}} \omega_{5}^{0}\left(0, U^{\dagger} d U\right) \\
& -c^{\prime} \int_{S^{4}}\left[\rho_{4}\left(0, U^{\dagger} d U, R\right)+\rho_{4}\left(0, L, U(R+d) U^{\dagger}\right)\right]
\end{aligned}
$$

This is nothing but the gauged Wess-Zumino-Witten action in the left-right symmetric regularization scheme $[41,42]$. (Naturally, the role of the electroweak fields in the standard action is played by the hadronic vector and axial-vector field here.) The effective anomalous action (2.105) can therefore be thought of as a generalization of the standard gauged Wess-Zumino-Witten action in that it contains two extra dynamical fields which work to compensate potentially dangerous gauge anomaly belonging to $\left[U(n)_{L} \times U(n)_{R}\right]^{(H L S)}$.

\section{$3 \quad$ Effective action with electroweak coupling}

In the previous section, we have derived from the extended NJL model an effective meson lagrangian containing not only Goldstone bosons but also hadronic vector and axial-vector 
mesons. The obtained effective action, including the intrinsic-parity nonconserving part, is shown to have the $\left[U(n)_{L} \times U(n)_{R}\right]_{\text {local }}^{(H L S)}$ symmetry as well as the $\left[U(n)_{L} \times U(n)_{R}\right]_{\text {global }}^{(e x t)}$ symmetry. Since the enlarged hidden local symmetry is completely maintained even at the quantum level, the hadronic vector and axial-vector mesons in this effective lagrangian can be regarded as gauge bosons of this symmetry. On the other hand, the global chiral symmetry possessed by the original extended NJL lagrangian is maintained by choosing the left-right symmetric form of anomaly under formally enlarged local symmetry belonging to $\left[U(n)_{L} \times U(n)_{R}\right]^{(e x t)}$. This choice is physically a natural one, since it respects the global chiral symmetry, a fundamental symmetry of strong interactions. So far, everything goes well without any trouble. However, once the couplings with the external electroweak fields is introduced, a nontrivial problem arises. In this section, we shall explain this problem together with its possible resolution by paying special attention to the role of the hidden local symmetry in our effective lagrangian.

The electroweak interactions can be introduced into the extended NJL model through the standard minimal replacement in the quark kinetic part of the lagrangian $(*)$ :

$$
\bar{q} i \gamma^{\mu} \partial_{\mu} q \longrightarrow \bar{q} i \gamma^{\mu}\left(\partial_{\mu}+l_{\mu} P_{L}+r_{\mu} P_{R}\right) q+\mathcal{L}_{\text {gauge }}
$$

where $\mathcal{L}_{\text {gauge }}$ represents the lagrangian of $S U(2)_{L} \times U(1)$ electroweak gauge theory, which contains kinetic terms of the external gauge fields and Higgs fields etc. (We shall omit $\mathcal{L}_{\text {gauge }}$ in the following expressions.) The external gauge fields $l_{\mu}$ and $r_{\mu}$ are expressed in terms of the photon $\left(\mathcal{B}_{\mu}\right)$ and weak bosons $\left(\mathcal{W}_{\mu}^{ \pm}\right.$and $\left.\mathcal{Z}_{\mu}^{0}\right)$ as

$$
\begin{aligned}
l_{\mu}= & i e Q\left(\mathcal{B}_{\mu}-\tan \theta_{W} \mathcal{Z}_{\mu}^{0}\right) \\
& +i \frac{e}{\sin \theta_{W} \cos \theta_{W}} T^{3} \mathcal{Z}_{\mu}^{0}+i \frac{e}{\sqrt{2} \sin \theta_{W}} \mathcal{C} \mathcal{W}_{\mu}
\end{aligned}
$$




$$
r_{\mu}=i e Q\left(\mathcal{B}_{\mu}-\tan \theta_{W} \mathcal{Z}_{\mu}^{0}\right)
$$

where $\theta_{W}, T^{3}$ and $Q$ are respectively the Weinberg angle, the third component of weak isospin and the electric charge. Finally, $C$ is the generalized Cabbibo matrix.

We can now proceed along the same line as described in sect.2. First introduce the collective meson field through the addition of (2.3). Then, instead of (2.6), we obtain

$$
\begin{aligned}
\mathcal{L}=\bar{q} & {\left[i \gamma^{\mu}\left\{\partial_{\mu}+\left(L_{\mu}+l_{\mu}\right) P_{L}+\left(R_{\mu}+r_{\mu}\right) P_{R}\right\}-\left(M^{\dagger} P_{L}+M P_{R}\right)\right] q } \\
& -\frac{1}{4 G_{S}} \operatorname{tr} M M^{\dagger}-\frac{1}{8 G_{V}} \operatorname{tr}\left[L_{\mu}^{2}+R_{\mu}^{2}\right] .
\end{aligned}
$$

Again redefining the quark fields through the chiral rotation (2.23), we obtain

$$
\begin{aligned}
& \bar{q} i \gamma^{\mu}\left\{\partial_{\mu}+\left(L_{\mu}+l_{\mu}\right) P_{L}+\left(R_{\mu}+r_{\mu}\right) P_{R}\right\} q \\
= & \bar{\chi}_{L} i \gamma^{\mu}\left\{\partial_{\mu}+\xi_{L}\left(\partial_{\mu}+L_{\mu}+l_{\mu}\right) \xi_{L}^{\dagger}\right\} \chi_{L} \\
= & \bar{\chi}_{R} i \gamma^{\mu}\left\{\partial_{\mu}+\xi_{R}\left(\partial_{\mu}+R_{\mu}+r_{\mu}\right) \xi_{R}^{\dagger}\right\} \chi_{R} .
\end{aligned}
$$

Since $L_{\mu}$ and $l_{\mu}\left(R_{\mu}\right.$ and $\left.r_{\mu}\right)$ appear here in the form $L_{\mu}+l_{\mu}\left(R_{\mu}+r_{\mu}\right)$, we find it convenient to introduce the following redefinition :

$$
\begin{aligned}
\mathcal{L}_{\mu} & =L_{\mu}+l_{\mu}, \\
\mathcal{R}_{\mu} & =R_{\mu}+r_{\mu} .
\end{aligned}
$$

We also introduce chirally rotated fields of $\mathcal{L}_{\mu}$ and $\mathcal{R}_{\mu}$ by

$$
\begin{aligned}
& \tilde{\mathcal{L}}_{\mu}=\xi_{L}\left(\mathcal{L}_{\mu}+\partial_{\mu}\right) \xi_{L}^{\dagger}, \\
& \tilde{\mathcal{R}}_{\mu}=\xi_{R}\left(\mathcal{R}_{\mu}+\partial_{\mu}\right) \xi_{R}^{\dagger},
\end{aligned}
$$

Now using these new variables together with the previously introduced representations (2.8) 
and (2.15) with $H \simeq<H>=m,(3.4)$ can be recast into the form :

$$
\begin{aligned}
\mathcal{L}= & \chi\left[i \gamma^{\mu}\left(\partial_{\mu}+\tilde{\mathcal{L}}_{\mu} P_{L}+\tilde{\mathcal{R}}_{\mu} P_{R}\right)-m\left(\xi_{M}^{\dagger} P_{L}+\xi_{M} P_{R}\right)\right] \chi \\
& -\frac{1}{8 G_{V}} \operatorname{tr}\left[\tilde{\mathcal{L}}_{\mu}+\partial_{\mu} \xi_{L} \xi_{L}^{\dagger}-\xi_{L} l_{\mu} \xi_{L}^{\dagger}\right]^{2} \\
& -\frac{1}{8 G_{V}} \operatorname{tr}\left[\tilde{\mathcal{R}}_{\mu}+\partial_{\mu} \xi_{R} \xi_{R}^{\dagger}-\xi_{R} r_{\mu} \xi_{R}^{\dagger}\right]^{2} .
\end{aligned}
$$

After performing an approximate bosonisation procedure just as before, we are then led to the following effective lagrangian for the nonanomalous part :

$$
\begin{aligned}
\mathcal{L}^{(n)}= & \frac{1}{4 g_{V}^{2}} \operatorname{tr}\left[\tilde{\mathcal{L}}_{\mu \nu}^{2}+\tilde{\mathcal{R}}_{\mu \nu}^{2}\right]-\frac{a}{a-1} \frac{f_{\pi}^{2}}{4} \operatorname{tr}\left[D_{\mu} \xi_{M} \cdot \xi_{M}^{\dagger}\right]^{2} \\
& -\frac{1}{2} a f_{\pi}^{2} \operatorname{tr}\left[D_{\mu} \xi_{L} \cdot \xi_{L}^{\dagger}\right]^{2}-\frac{1}{2} a f_{\pi}^{2} \operatorname{tr}\left[D_{\mu} \xi_{R} \cdot \xi_{R}^{\dagger}\right]^{2} .
\end{aligned}
$$

where

$$
\begin{aligned}
& \tilde{\mathcal{L}}_{\mu \nu}=\partial_{\mu} \tilde{\mathcal{L}}_{\nu}-\partial_{\nu} \tilde{\mathcal{L}}_{\mu}+\left[\tilde{\mathcal{L}}_{\mu}, \tilde{\mathcal{L}}_{\nu}\right] \\
& \tilde{\mathcal{R}}_{\mu \nu}=\partial_{\mu} \tilde{\mathcal{R}}_{\nu}-\partial_{\nu} \tilde{\mathcal{R}}_{\mu}+\left[\tilde{\mathcal{R}}_{\mu}, \tilde{\mathcal{R}}_{\nu}\right]
\end{aligned}
$$

and

$$
\begin{aligned}
D_{\mu} \xi_{L} & =\partial_{\mu} \xi_{L}+\tilde{\mathcal{L}}_{\mu} \xi_{L}-\xi_{L} l_{\mu} \\
D_{\mu} \xi_{R} & =\partial_{\mu} \xi_{R}+\tilde{\mathcal{R}}_{\mu} \xi_{R}-\xi_{R} r_{\mu} \\
D_{\mu} \xi_{M} & =\partial_{\mu} \xi_{M}+\tilde{\mathcal{L}}_{\mu} \xi_{M}-\xi_{M} \tilde{\mathcal{R}}_{\mu}
\end{aligned}
$$

Identifying $\tilde{\mathcal{L}}_{\mu}$ and $\tilde{\mathcal{R}}_{\mu}$ as the gauge bosons of the enlarged hidden local symmetry, the above lagrangian precisely coincides with the corresponding lagrangian of Bando et al. with the electroweak couplings except that the arbitrary constants $a^{\prime}, b^{\prime}, c^{\prime}, d^{\prime}$ of their model are again constrained such that $a^{\prime}=b^{\prime}=a, c^{\prime}=a /(a-1)$ and $d^{\prime}=0$ in our effective lagrangian. As 
already discussed by Bando et al. [6], an especially interesting gauge corresponds to taking $\xi_{L}=\xi_{R}=1, \xi_{M}=U$. In this particular gauge, (3.11) reduces to

$$
\begin{aligned}
\mathcal{L}^{(n)}= & \frac{1}{4 g_{V}^{2}} \operatorname{tr}\left[\mathcal{L}_{\mu \nu}^{2}+\mathcal{R}_{\mu \nu}^{2}\right]+\frac{a}{a-1} \frac{f_{\pi}^{2}}{4} \operatorname{tr}\left(D_{\mu} U D^{\mu} U^{\dagger}\right) \\
& -\frac{1}{2} a f_{\pi}^{2} \operatorname{tr}\left(\mathcal{L}_{\mu}-l_{\mu}\right)^{2}-\frac{1}{2} a f_{\pi}^{2} \operatorname{tr}\left(\mathcal{R}_{\mu}-r_{\mu}\right)^{2}
\end{aligned}
$$

with

$$
\begin{gathered}
D_{\mu} U \equiv \partial_{\mu} U+\mathcal{L}_{\mu} U-U \mathcal{R}_{\mu} \\
D_{\mu} U^{\dagger} \equiv \partial_{\mu} U^{\dagger}-U^{\dagger} \mathcal{L}_{\mu}+\mathcal{R}_{\mu} U^{\dagger}
\end{gathered}
$$

Identifying $\mathcal{L}_{\mu}$ and $\mathcal{R}_{\mu}$ as physical vector and axial-vector mesons, (3.17) is essentially the lagrangian of the massive Yang-Mills model supplemented by the VMD-type direct couplings between the hadronic vector and axial-vector mesons and the external gauge fields $l_{\mu}$ and $r_{\mu}$ $[1,2]$

Now we turn to more interesting anomalous part of the action. For a pedagogical reason, we first show how the naive quantization procedure cause a trouble, and then explain how the trouble can be circumvented following the recent proposal by Bijinens and Prades and by Arriola and Salcedo. As is clear from the discussion in sect.2, the fermion path integral $Z_{f}$ can be written in the following forms :

$$
\begin{aligned}
Z_{f} & =\int \mathcal{D} q \mathcal{D} \bar{q} e^{i \int d^{4} x \bar{q} \mathcal{D} q} \\
& =J_{1}^{N_{c}} \int \mathcal{D} \chi \mathcal{D} \bar{\chi} e^{i \int d^{4} x \bar{\chi} \tilde{\mathcal{D}} \chi} \\
& =J_{1}^{N_{c}} J_{2}{ }^{N_{c}} \int \mathcal{D} \varphi \mathcal{D} \bar{\varphi} e^{i \int d^{4} x \bar{\varphi} \hat{\mathcal{D}} \varphi}
\end{aligned}
$$

where

$$
\mathcal{D}=i \gamma^{\mu}\left(\partial_{\mu}+\mathcal{L}_{\mu} P_{L}+\mathcal{R}_{\mu} P_{R}\right)-m\left(\xi_{R}^{\dagger} \xi_{M}^{\dagger} \xi_{L} P_{L}+\xi_{L}^{\dagger} \xi_{M} \xi_{R} P_{R}\right)
$$




$$
\begin{aligned}
& \tilde{\mathcal{D}}=i \gamma^{\mu}\left(\partial_{\mu}+\tilde{\mathcal{L}}_{\mu} P_{L}+\tilde{\mathcal{R}}_{\mu} P_{R}\right)-m\left(\xi_{M}^{\dagger} P_{L}+\xi_{M} P_{R}\right), \\
& \hat{\mathcal{D}}=i \gamma^{\mu}\left(\partial_{\mu}+\hat{\mathcal{L}}_{\mu} P_{L}+\hat{\mathcal{R}}_{\mu} P_{R}\right)-m .
\end{aligned}
$$

which have the same forms as $(2.37) \sim(2.40)$ except that $L_{\mu}$ and $R_{\mu}$ and their chirally rotated fields $\tilde{L}_{\mu}, \tilde{R}_{\mu}, \hat{L}_{\mu}, \hat{R}_{\mu}$ there are now replaced by $\mathcal{L}_{\mu} \equiv L_{\mu}+l_{\mu}, \mathcal{R}_{\mu} \equiv R_{\mu}+r_{\mu}$ and their chirally rotated correspondents. It is therefore quite natural to think that the anomalous action with the external electroweak couplings is obtained from (2.60) in the previous section simply by replacing $L_{\mu}, R_{\mu}, \tilde{L}_{\mu}, \tilde{R}_{\mu}, \hat{L}_{\mu}, \hat{R}_{\mu}$ by $\mathcal{L}_{\mu}, \mathcal{R}_{\mu}, \tilde{\mathcal{L}}_{\mu}, \tilde{\mathcal{R}}_{\mu}, \hat{\mathcal{L}}_{\mu}, \hat{\mathcal{R}}_{\mu}$. However, it turns out that this simplest construction does not meets the requirement of QCD phenomenology. To see it, we first recall the symmetries possessed by such an action. Its symmetries are $\left[U(n)_{L} \times U(n)_{R}\right]_{\text {global }}^{(e x t)} \times\left[U(n)_{L} \times U(n)_{R}\right]^{(H L S)}$. Remember that the global chiral symmetry here is the consequence of our choice of the left-right symmetric regularization scheme. In the absence of the electroweak couplings, this choice has nothing to be questioned, since it respects the global chiral symmetry, i.e. the fundamental symmetry of strong interactions. However, since the electromagnetic gauge group is now contained in the diagonal subgroup $\left[U(n)_{V}\right]^{(e x t)}$ of $\left[U(n)_{L} \times U(n)_{R}\right]^{(e x t)}$, the anomalous action in the left-right symmetric regularization scheme breaks electromagnetic gauge invariance. This is nothing but the problem several authors had encountered when trying to construct the anomalous action based on the idea of gauging the external chiral symmetry [28-31]. To recover the electromagnetic gauge invariance, they then decided to adopt the vector-gauge invariant regularization scheme, which is attained by subtracting a local counter term (called the Bardeen subtraction) depending on the hadronic vector and axial-vector fields. This cannot get rid of the trouble, however. The gauged Wess-Zumino-Witten action in the vector-gauge invariant scheme inevitably breaks 
the global chiral symmetry at the strong interaction level in the present setting. Consequently, the famous low-energy theorem for the purely hadronic process $K^{+} K^{-} \longrightarrow 3 \pi$ is not correctly reproduced. It was also shown [22] that the above symmetry violation, in combination with the mixing of the Goldstone boson and the axial-vector field, brings about theoretically unpleasant correction to the process $\gamma \longrightarrow 3 \pi$. It seemed that there is no way out of this dilemma, considering that the anomaly can be shifted from one place to another but it cannot be eliminate completely.

However, here is a pitfall. It was an implicit assumption of the argument so far that the local counter terms in this anomaly shifting procedure are function of $\mathcal{L}_{\mu}$ and $\mathcal{R}_{\mu}$ only. As has been pointed out by Bijinens and Prades quite recently, this may not be necessarily true [32]. According to them, this corresponds to the standardly used procedure in which the functional measure of the hadronic vector and axial-vector fields is defined by the Dirac operator (3.21) that is a function of $\mathcal{L}_{\mu}$ and $\mathcal{R}_{\mu}$ rather than a function of $L_{\mu}$ and $R_{\mu}$, which has no a priori justification. This observation opens up a possibility to use more general renormalization procedure. That is one is now allowed to subtract local counter terms, which has general dependence on $L_{\mu}$ and $l_{\mu}$ (and $R_{\mu}$ and $r_{\mu}$ ), in the construction of the effective meson action. Recently, Arriola and Salcedo has made use of this observation for explicitly constructing the anomalous action with the required symmetries [33]. Here we shall carry out a similar construction in our scheme with the extra hidden local symmetry.

We start with the anomalous action :

$$
\begin{aligned}
\Gamma_{L R}^{(a)} & =c^{\prime} \int_{B^{5}}\left[\omega_{5}^{0}\left(0, \xi_{R}^{\dagger} d \xi_{R}\right)-\omega_{5}^{0}\left(0, \xi_{L}^{\dagger} d \xi_{L}\right)-\omega_{5}^{0}\left(0, \xi_{M} d \xi_{M}^{\dagger}\right)\right] \\
& +c^{\prime} \int_{S^{4}}\left[\rho_{4}\left(0, \mathcal{R}, \xi_{R}^{\dagger} d \xi_{R}\right)+\rho_{4}\left(0, \xi_{L}^{\dagger} d \xi_{L}, \mathcal{L}\right)\right.
\end{aligned}
$$




$$
\left.+\rho_{4}\left(0, \xi_{M} d \xi_{M}^{\dagger}, \tilde{\mathcal{L}}\right)-\rho_{4}(0, \hat{\mathcal{L}}, \hat{\mathcal{R}})\right]
$$

which is obtained from (2.104) simply by replacing $L_{\mu}, R_{\mu}, \tilde{L}_{\mu}, \tilde{R}_{\mu}, \hat{L}_{\mu}, \hat{R}_{\mu}$ by $\mathcal{L}_{\mu}, \mathcal{R}_{\mu}, \tilde{\mathcal{L}}_{\mu}$ $\tilde{\mathcal{R}}_{\mu}, \hat{\mathcal{L}}_{\mu}, \hat{\mathcal{R}}_{\mu}$. As will be shown in Appendix A, this effective action has the following anomaly structure :

$$
\begin{aligned}
\delta_{L}^{(e x t)} \Gamma_{L R}^{(a)} & =G_{L}\left(\theta_{L} ; \mathcal{L}, \mathcal{R}\right)=c^{\prime} \int_{S^{4}} \bar{\Delta}\left(\theta_{L}, \mathcal{L}\right), \\
\delta_{R}^{(e x t)} \Gamma_{L R}^{(a)} & =G_{R}\left(\theta_{R} ; \mathcal{L}, \mathcal{R}\right)=-c^{\prime} \int_{S^{4}} \bar{\Delta}\left(\theta_{R}, \mathcal{R}\right), \\
\delta_{L}^{(H L S)} \Gamma_{L R}^{(a)} & =\delta_{R}^{(H L S)} \Gamma_{L R}^{(a)}=0 .
\end{aligned}
$$

We shall see below that allowing general counter terms, which depends on $\mathcal{L}_{\mu}$ and $r_{\mu}$ (and $\mathcal{R}_{\mu}$ and $l_{\mu}$ ), one can shift the anomaly to the external electroweak sector. Before doing this, we should add two remarks, which would help to avoid confusion. First, it is important to recognize the fact that in the effective action $\Gamma_{L R}^{(a)}$ the anomaly resides only in the $\left[U(n)_{L} \times U(n)_{R}\right]^{(e x t)}$ group, and the $\left[U(n)_{L} \times U(n)_{R}\right]^{(H L S)}$ group is anomaly free. The subtraction of local counter terms, which depends on $\mathcal{L}_{\mu}$ and $\mathcal{R}_{\mu}$, does not change the anomalyfree nature of the $\left[U(n)_{L} \times U(n)_{R}\right]^{(H L S)}$ group, since $\mathcal{L}_{\mu}$ and $\mathcal{R}_{\mu}$ are absolutely intact under this group transformations. Secondly, as counter terms, we choose a function of $\mathcal{L}_{\mu}$ and $l_{\mu}$ (and $\mathcal{R}_{\mu}$ and $r_{\mu}$ ) instead of the choice in [33], where it is chosen as a function of $L_{\mu}$ and $l_{\mu}$ (and $R_{\mu}$ and $r_{\mu}$ ), where $\mathcal{L}_{\mu}=L_{\mu}+l_{\mu}$ (and $\mathcal{R}_{\mu}=R_{\mu}+r_{\mu}$ ). This however makes no essential difference, since a function of $L_{\mu}$ and $l_{\mu}$ can trivially be expressed as (another) function of $\mathcal{L}_{\mu}=L_{\mu}+l_{\mu}$ and $l_{\mu}$. Our choice here is motivated by the fact that not $L_{\mu}$ and $R_{\mu}$ but $\mathcal{L}_{\mu}$ and $\mathcal{R}_{\mu}$ (or $\tilde{\mathcal{L}}_{\mu}$ and $\tilde{\mathcal{R}}_{\mu}$ ) should be identified with the physical fields in order to eliminate the kinetic term mixing resulting from the bosonisation of (3.4).

Now we define the new action from (3.24) by subtracting an appropriate local counter 
term as

$$
\bar{\Gamma}^{(a)} \equiv \Gamma_{L R}^{(a)}-\Gamma_{\text {c.t. }}^{(a)}
$$

Here we require that the counter term is globally chiral invariant, so that the subtraction of it preserves this symmetry. There are eight globally chiral invariant pieces with dimension four, which can be constructed from $\mathcal{L}$ and $l$. They are

$$
\begin{aligned}
& \operatorname{tr}\left[\mathcal{L}^{3} l\right], \quad \operatorname{tr}\left[\mathcal{L}^{2} l^{2}\right], \quad \operatorname{tr}[\mathcal{L} l \mathcal{L} l], \quad \operatorname{tr}\left[\mathcal{L} l^{3}\right], \\
& \operatorname{tr}[d \mathcal{L} \mathcal{L} l], \operatorname{tr}[\mathcal{L} d \mathcal{L} l], \quad \operatorname{tr}[\mathcal{L} d l l], \quad \operatorname{tr}[\mathcal{L} l d l] .
\end{aligned}
$$

We then rewrite $\Gamma_{c . t .}^{(a)}$ as a linear combination of these quantities and their right-handed counterparts as

$$
\begin{aligned}
& \Gamma_{c . t .}^{(a)}=- \frac{c^{\prime}}{2} \int_{S^{4}} \operatorname{tr}\left[c_{1} \mathcal{L} l^{3}+c_{2} \mathcal{L} d l l+c_{3} \mathcal{L} l d l+c_{4} \mathcal{L}^{2} l^{2}\right. \\
&\left.\quad+c_{5} \mathcal{L} l \mathcal{L} l+c_{6} d \mathcal{L} \mathcal{L} l+c_{7} \mathcal{L} d \mathcal{L} l+c_{8} \mathcal{L}^{3} l\right] \\
& \quad-\quad(\mathcal{L} \leftrightarrow \mathcal{R}, l \leftrightarrow r) .
\end{aligned}
$$

The eight unknown coefficients $c_{1}, \cdots, c_{8}$ can be determined such that the new action $\bar{\Gamma}^{(a)}$ satisfies the following conditions :

$$
\begin{aligned}
\delta_{L}^{(e x t)} \bar{\Gamma}^{(a)} & =G_{L}\left(\theta_{L} ; l, r\right), \\
\delta_{R}^{(e x t)} \bar{\Gamma}^{(a)} & =G_{R}\left(\theta_{L} ; l, r\right), \\
\delta_{L}^{(H L S)} \bar{\Gamma}^{(a)} & =\delta_{R}^{(H L S)} \bar{\Gamma}^{(a)}=0 .
\end{aligned}
$$

Here, the third condition is trivially satisfied, since the subtracted counter term is hidden gauge invariant. The first and the second conditions can alternatively be expressed as

$$
\delta_{L}^{(e x t)} \Gamma_{\text {c.t. }}^{(a)}=G_{L}\left(\theta_{L} ; \mathcal{L}, \mathcal{R}\right)-G_{L}\left(\theta_{L} ; l, r\right)
$$




$$
\delta_{R}^{(e x t)} \Gamma_{c . t .}^{(a)}=G_{R}\left(\theta_{R} ; \mathcal{L}, \mathcal{R}\right)-G_{R}\left(\theta_{R} ; l, r\right)
$$

which is fulfilled if one take as

$$
c_{1}=c_{2}=c_{3}=1, \quad c_{4}=0, \quad c_{5}=1 / 2, \quad c_{6}=c_{7}=c_{8}=1,
$$

which gives the desired counter term :

$$
\begin{aligned}
\Gamma_{c . t .}^{(a)}=-\frac{c^{\prime}}{2} \int_{S^{4}} & \operatorname{tr}\left[\mathcal{L} l^{3}+\mathcal{L}\{d l, l\}+\frac{1}{2} \mathcal{L} l \mathcal{L} l\right. \\
+ & \left.\{d \mathcal{L}, \mathcal{L}\} l+\mathcal{L}^{3} l\right]-(\mathcal{L} \leftrightarrow \mathcal{R}, l \leftrightarrow r) .
\end{aligned}
$$

Now one sees from (3.31) and (3.32) that the anomaly is totally shifted from the hadronic sector to the external electroweak sector. To recover the electromagnetic gauge invariance completely, we need further redefinition of the anomalous action as

$$
\Gamma^{(a)} \equiv \bar{\Gamma}^{(a)}-\Gamma_{L R}^{(a)}\left[\xi_{L}=\xi_{R}=\xi_{M}=1 ; l, r\right]
$$

where the subtracted term above is nothing but the familiar Bardeen subtraction, which depends on the external gauge fields $l$ and $r$ instead of the hadronic fields $\mathcal{L}$ and $\mathcal{R}$. Now, the anomaly structure of our final action is given by

$$
\begin{aligned}
\delta_{V}^{(e x t)} \Gamma^{(a)} & =0 \\
\delta_{A}^{(e x t)} \Gamma^{(a)} & =G_{B}\left(\theta_{R}=-\theta_{L}=\theta ; v, a\right), \\
\delta_{V}^{(H L S)} \Gamma^{(a)} & =\delta_{A}^{(H L S)} \Gamma^{(a)}=0 .
\end{aligned}
$$

As a matter of course, there still is an axial anomaly, which depends on the external electroweak fields. However, it is the standardly accepted scenario that it is to be canceled by the corresponding lepton loop contribution owing to the quark-lepton symmetry. Now our 
final action satisfies all the symmetries as required as an effective theory of QCD (except for $U_{A}(1)$ anomaly). For convenience, we summarize the final form of the effective action derived here from the extended NJL model with inclusion of the enlarged hidden gauge symmetry :

$$
\Gamma_{e f f}=\Gamma^{(n)}+\Gamma^{(a)}
$$

where $\Gamma^{(n)}=\int d^{4} x \mathcal{L}^{(n)}$ with

$$
\begin{aligned}
\mathcal{L}^{(n)}= & \frac{1}{4 g_{V}^{2}} \operatorname{tr}\left[\tilde{\mathcal{L}}_{\mu \nu}^{2}+\tilde{\mathcal{R}}_{\mu \nu}^{2}\right]-\frac{a}{a-1} \frac{f_{\pi}^{2}}{4} \operatorname{tr}\left[D_{\mu} \xi_{M} \cdot \xi_{M}^{\dagger}\right]^{2} \\
& -\frac{1}{2} a f_{\pi}^{2} \operatorname{tr}\left[D_{\mu} \xi_{L} \cdot \xi_{L}^{\dagger}\right]^{2}-\frac{1}{2} a f_{\pi}^{2} \operatorname{tr}\left[D_{\mu} \xi_{R} \cdot \xi_{R}^{\dagger}\right]^{2} .
\end{aligned}
$$

and

$$
\begin{aligned}
\Gamma^{(a)}= & \Gamma_{L R}^{(a)}\left[\xi_{L}, \xi_{R}, \xi_{M} ; \tilde{\mathcal{L}}, \tilde{\mathcal{R}}\right] \\
& -\Gamma_{\text {c.t. }}^{(a)}[\mathcal{L}, \mathcal{R} ; l, r]-\Gamma_{L R}^{(a)}\left[\xi_{L}=\xi_{R}=\xi_{M}=1 ; l, r\right] .
\end{aligned}
$$

Taking the special gauge $\xi_{L}=\xi_{M}=1, \xi_{R}=U, \Gamma_{L R}^{(a)}\left[\xi_{L}, \xi_{R}, \xi_{M} ; \tilde{L}, \tilde{R}\right]$ reduces to the standard gauged Wess-Zumino-Witten action in the LR scheme, i.e. $\Gamma_{L R}^{(W Z)}[U ; \mathcal{L}, \mathcal{R}]$ given in terms of the field variables $U, \mathcal{L}$ and $\mathcal{R}$, while $\Gamma_{L R}^{(a)}\left[\xi_{L}=\xi_{R}=\xi_{M}=1 ; l, r\right]$ is nothing different from $\Gamma_{L R}^{(W Z)}[U=1 ; l, r]$. The total action then becomes

$$
\begin{aligned}
\mathcal{L}^{(n)}= & \frac{1}{4 g_{V}^{2}}\left[\mathcal{L}_{\mu \nu}^{2}+\mathcal{R}_{\mu \nu}^{2}\right]+\frac{a}{a-1} \frac{f_{\pi}^{2}}{4} \operatorname{tr}\left(D_{\mu} U D^{\mu} U^{\dagger}\right) \\
& -\frac{1}{2} a f_{\pi}^{2} \operatorname{tr}\left(\mathcal{L}_{\mu}-l_{\mu}\right)^{2}-\frac{1}{2} a f_{\pi}^{2} \operatorname{tr}\left(\mathcal{R}_{\mu}-r_{\mu}\right)^{2},
\end{aligned}
$$

and

$$
\Gamma^{(a)}=\Gamma_{L R}^{(W Z)}[U ; \mathcal{L}, \mathcal{R}]-\Gamma_{\text {c.t. }}^{(a)}[\mathcal{L}, \mathcal{R} ; l, r]-\Gamma_{L R}^{(W Z)}[U=1 ; l, r]
$$

which essentially coincides with the result of Arriola and Salcedo except that we express the action in terms of $\mathcal{L}$ and $l(\mathcal{R}$ and $r)$, while they do in terms of $L=\mathcal{L}-l$ and $l(R=\mathcal{R}-r$ and $r$ ). 
It seems clear by now that the idea of the hidden local symmetry plays no positive role in the above construction of the phenomelologically consistent effective action. What is important from the physical viewpoint is response of the action under the external global or local variations. This seems reasonable because the structure of hadronic currents or their associated observables can be seen only with the external electroweak probes. In the following discussion on the theoretical structure of the hadronic currents, we therefore concentrate on the hidden gauge fixed version of the effective action, for convenience.

As first pointed out in [32], the aforementioned subtraction of the local counter term, which depends on $\mathcal{L}_{\mu}$ and $l_{\mu}$ (and $\mathcal{R}_{\mu}$ and $r_{\mu}$ ), modifies the vector meson dominance (VMD), which is otherwise exact in the extended NJL model. How it is modified can be seen as follows. To this end, we first divide the total effective action $\Gamma_{e f f}=\Gamma^{(n)}+\Gamma^{(n)}$ into purely hadronic part and the other part that consists of terms containing at least one external gauge fields :

$$
\Gamma_{\text {eff }}=\Gamma_{\text {strong }}+\text { terms containing electroweak fields }
$$

where $\Gamma_{\text {strong }}$ is given by

$$
\Gamma_{\text {strong }}=\Gamma_{f}-\frac{M_{V}^{2}}{2 g_{V}^{2}} \operatorname{tr}\left[\mathcal{L}_{\mu}^{2}+\mathcal{R}_{\mu}^{2}\right],
$$

with $\Gamma_{f}$ being the part, which comes from the path integral of the fermion determinant with appropriate counter term subtraction, i.e.

$$
\Gamma_{f} \equiv-\left.i N_{c} \log \operatorname{det} D(U, \mathcal{L}, \mathcal{R})\right|_{\text {renorm }}
$$

Our lowest order answer has been

$$
\begin{aligned}
\Gamma_{f}= & \frac{1}{4 g_{V}^{2}} \operatorname{tr}\left[\mathcal{L}_{\mu \nu}^{2}+\mathcal{R}_{\mu \nu}^{2}\right]+\frac{a}{a-1} \frac{f_{\pi}^{2}}{4} \operatorname{tr}\left(D_{\mu} U D^{\mu} U^{\dagger}\right) \\
& +\Gamma_{L R}^{(W Z)}[U ; \mathcal{L}, \mathcal{R}]-\Gamma_{\text {c.t. }}^{(a)}[\mathcal{L}, \mathcal{R} ; l, r]-\Gamma_{L R}^{(W Z)}[U=1 ; l, r]
\end{aligned}
$$


Now we consider the change of $\Gamma_{\text {strong }}$ under an infinitesimal left variation, which gives

$$
\begin{aligned}
\delta_{L}^{(e x t)} \Gamma_{\text {strong }} & =\int \operatorname{tr}\left\{\delta_{L}^{(e x t)} \mathcal{L}_{\mu} \frac{\delta \Gamma_{\text {strong }}}{\delta \mathcal{L}_{\mu}}+\delta_{L}^{(e x t)} U \frac{\delta \Gamma_{\text {strong }}}{\delta U}\right\} \\
& =\int \operatorname{tr}\left\{\delta_{L}^{(e x t)} \mathcal{L}_{\mu}\left(\frac{\delta \Gamma_{f}}{\delta \mathcal{L}_{\mu}}-\frac{M_{V}^{2}}{g_{V}^{2}} \mathcal{L}_{\mu}\right)+\delta_{L}^{(e x t)} U \frac{\delta \Gamma_{\text {strong }}}{\delta U}\right\} \\
& =\int \operatorname{tr} \theta_{L}\left\{-D^{\mu}(\mathcal{L}) \frac{\delta \Gamma_{f}}{\delta \mathcal{L}_{\mu}}+\frac{M_{V}^{2}}{g_{V}^{2}} \partial^{\mu} \mathcal{L}_{\mu}-U \frac{\delta \Gamma_{\text {strong }}}{\delta U}\right\}
\end{aligned}
$$

Here we have performed a partial integration and introduced the covariant derivative $D^{\mu}(\mathcal{L})$ operating on a matrix $M$ by

$$
D^{\mu}(\mathcal{L}) M=\partial^{\mu} M+\left[\mathcal{L}^{\mu}, M\right] .
$$

Using the equation of motion for the Goldstone field, i.e. $\delta \Gamma_{\text {strong }} / \delta U=0$, we then obtain

$$
\delta_{L}^{(e x t)} \Gamma_{\text {strong }}=-\int \operatorname{tr} \theta_{L}\left\{D^{\mu}(\mathcal{L}) j_{\mu}^{L}-\frac{M_{V}^{2}}{g_{V}^{2}} \partial^{\mu} \mathcal{L}_{\mu}\right\}
$$

where

$$
j_{\mu}^{L} \equiv \frac{\delta}{\delta \mathcal{L}_{\mu}} \Gamma_{f}
$$

is the basic quark left-hand current (or more precisely its bosonic equivalent). Similarly, the infinitesimal right variation of $\Gamma_{\text {strong }}$ gives

$$
\delta_{R}^{(e x t)} \Gamma_{\text {strong }}=-\int \operatorname{tr} \theta_{R}\left\{D^{\mu}(\mathcal{R}) j_{\mu}^{R}-\frac{M_{V}^{2}}{g_{V}^{2}} \partial^{\mu} \mathcal{R}_{\mu}\right\}
$$

with

$$
j_{\mu}^{R} \equiv \frac{\delta}{\delta \mathcal{R}_{\mu}} \Gamma_{f}
$$

Here, an important observation is as follows. The equation of motions for the hadronic fields result from the stationary requirement of $\Gamma_{\text {strong }}$ under arbitrary variations of $\mathcal{L}_{\mu}, \mathcal{R}_{\mu}$, and $U$. 
Since the gauge variations (3.53) and (3.55) are special cases of such arbitrary variations, it immediately follows that

$$
\delta_{L}^{(e x t)} \Gamma_{\text {strong }}=\delta_{R}^{(e x t)} \Gamma_{\text {strong }}=0
$$

Combining (3.54) and (3.55) and (3.57), we therefore obtain

$$
\begin{aligned}
D^{\mu}(\mathcal{L}) j_{\mu}^{L} & =\frac{M_{V}^{2}}{g_{V}^{2}} \partial^{\mu} \mathcal{L}_{\mu} \\
D^{\mu}(\mathcal{R}) j_{\mu}^{R} & =\frac{M_{V}^{2}}{g_{V}^{2}} \partial^{\mu} \mathcal{R}_{\mu} .
\end{aligned}
$$

Incidentally, the covariant derivatives of the basic currents give anomaly (it can be easily verified by carrying out a similar manipulation as above for $\Gamma_{f}$ instead of $\Gamma_{\text {strong }}$ ) as

$$
\begin{aligned}
D^{\mu}(\mathcal{L}) j_{\mu}^{L} & =-\partial^{\mu} \Delta_{\mu}\left(\mathcal{L}_{\mu}\right) \\
D^{\mu}(\mathcal{R}) j_{\mu}^{R} & =\partial^{\mu} \Delta_{\mu}\left(\mathcal{R}_{\mu}\right)
\end{aligned}
$$

with

$$
\begin{aligned}
\Delta^{\mu}\left(\mathcal{L}_{\mu}\right) & =-\frac{c^{\prime}}{2} \varepsilon^{\mu \nu \rho \sigma}\left[\left\{\mathcal{L}_{\nu}, \partial_{\rho} \mathcal{L}_{\sigma}\right\}+\mathcal{L}_{\nu} \mathcal{L}_{\rho} \mathcal{L}_{\sigma}\right] \\
\Delta^{\mu}\left(\mathcal{R}_{\mu}\right) & =-\frac{c^{\prime}}{2} \varepsilon^{\mu \nu \rho \sigma}\left[\left\{\mathcal{R}_{\nu}, \partial_{\rho} \mathcal{R}_{\sigma}\right\}+\mathcal{R}_{\nu} \mathcal{R}_{\rho} \mathcal{R}_{\sigma}\right]
\end{aligned}
$$

Remember that the total derivative nature of the anomaly results from our choice of the left-right symmetric form of anomaly. Combining (3.58),(3.59), and (3.60),(3.61), we then find

$$
\begin{aligned}
& \partial^{\mu}\left(\frac{M_{V}^{2}}{g_{V}^{2}} \mathcal{L}_{\mu}+\Delta_{\mu}\left(\mathcal{L}_{\mu}\right)\right)=0 \\
& \partial^{\mu}\left(\frac{M_{V}^{2}}{g_{V}^{2}} \mathcal{R}_{\mu}-\Delta_{\mu}\left(\mathcal{R}_{\mu}\right)\right)=0
\end{aligned}
$$


which insists the existence of the conserved currents given by

$$
\begin{aligned}
\bar{J}_{\mu}^{L} & \equiv \frac{M_{V}^{2}}{g_{V}^{2}} \mathcal{L}_{\mu}+\Delta_{\mu}\left(\mathcal{L}_{\mu}\right), \\
\bar{J}_{\mu}^{R} & \equiv \frac{M_{V}^{2}}{g_{V}^{2}} \mathcal{R}_{\mu}-\Delta_{\mu}\left(\mathcal{R}_{\mu}\right) .
\end{aligned}
$$

The existence of conserved currents was naturally expected from the fact that the left-right symmetric form of regularization preserves global chiral symmetry, as pointed out in [22]. However, the trouble observed in [22] was that these currents cannot be identified with the currents probed by the external electroweak gauge fields. This is related to the fact that the effective lagrangian in [22] with the left-right symmetric regularization scheme, breaks electromagnetic gauge invariance. In our present effective lagrangian, this trouble has now been remedied owing to the function of the newly subtracted local counter term. In fact, the hadronic electroweak currents are defined by

$$
\begin{aligned}
J_{\mu}^{L} & \left.\equiv \frac{\delta}{\delta l^{\mu}} \Gamma_{e f f}\right|_{l_{\mu}, r_{\mu} \longrightarrow 0}, \\
J_{\mu}^{R} & \left.\equiv \frac{\delta}{\delta r^{\mu}} \Gamma_{e f f}\right|_{l_{\mu}, r_{\mu} \longrightarrow 0} .
\end{aligned}
$$

Here, $\Gamma_{\text {eff }}$ is our total effective action (in a special gauge) given as

$$
\Gamma=\Gamma^{(n)}+\Gamma^{(a)},
$$

where

$$
\begin{aligned}
\Gamma^{(n)}= & -i N_{c} \log \operatorname{det}|D(U ; \mathcal{L}, \mathcal{R})| \\
& -\int d^{4} x \frac{M_{V}^{2}}{2 g_{V}^{2}} \operatorname{tr}\left[\left(\mathcal{L}_{\mu}-l_{\mu}\right)^{2}+\left(\mathcal{R}_{\mu}-r_{\mu}\right)^{2}\right] \\
\Gamma^{(a)}= & \Gamma_{L R}^{(W Z)}[U ; \mathcal{L}, \mathcal{R}]-\Gamma_{\text {c.t. }}^{(a)}[\mathcal{L}, \mathcal{R} ; l, r]-\Gamma_{L R}^{(W Z)}[U=1 ; l, r],
\end{aligned}
$$


with

$$
\begin{aligned}
& \Gamma_{c . t .}^{(a)}[\mathcal{L}, \mathcal{R} ; l, r]=-\frac{c^{\prime}}{2} \int_{S^{4}} \operatorname{tr}\left[\mathcal{L} l^{3}+\mathcal{L}\{d l, l\}+\frac{1}{2} \mathcal{L} l \mathcal{L} l\right. \\
&\left.+\{d \mathcal{L}, \mathcal{L}\} l+\mathcal{L}^{3} l\right]-(\mathcal{L} \leftrightarrow \mathcal{R}, l \leftrightarrow r), \\
& \Gamma_{L R}^{(W Z)}[U=1 ; l, r]=-\frac{c^{\prime}}{2} \int_{S^{4}} \operatorname{tr}\left[(l r-r l)\left(\mathcal{F}_{l}+\mathcal{F}_{r}\right)\right. \\
&\left.-l^{3} r+r^{3} l+\frac{1}{2} l r l r\right],
\end{aligned}
$$

with $\mathcal{F}_{l}=d l+l^{2}, \mathcal{F}_{r}=d r+r^{2}$. Performing the functional derivative on $l^{\mu}$ and $r^{\mu}$ and then letting $l_{\mu}$ and $r_{\mu}$ be zero, we find that

$$
\begin{aligned}
J_{\mu}^{L} & \equiv \frac{M_{V}^{2}}{g_{V}^{2}} \mathcal{L}_{\mu}+\Delta_{\mu}\left(\mathcal{L}_{\mu}\right) \\
J_{\mu}^{R} & \equiv \frac{M_{V}^{2}}{g_{V}^{2}} \mathcal{R}_{\mu}-\Delta_{\mu}\left(\mathcal{R}_{\mu}\right)
\end{aligned}
$$

which precisely coincide with $\bar{J}_{\mu}^{L}$ and $\bar{J}_{\mu}^{R}$, the conservation of which we have already proved. We are thus led to complete CVC and CAC relations as follows :

$$
\partial^{\mu} J_{\mu}^{V}=0, \quad \partial^{\mu} J_{\mu}^{A}=0
$$

with the definition $J_{\mu}^{V}=\frac{1}{2}\left(J_{\mu}^{R}+J_{\mu}^{L}\right)$ and $J_{\mu}^{A}=\frac{1}{2}\left(J_{\mu}^{R}-J_{\mu}^{L}\right)$. Eqs.(3.75) and (3.76) shows, as first pointed out by Bijnens and Prades [32], that the exact current-field identity is lost in the new scheme. Note however that it is only minimally modified. Since the deviation from the current-field identity depends on the vector and axial-vector fields only, the external electroweak gauge fields are coupled to the Goldstone bosons only through the hadronic vector and axial-vector fields. The vector (and axial-vector) meson dominance still holds in this narrow sense. 


\section{Summary and Discussion}

Using the standard auxiliary field method, we have derived from the extended NJL model an effective meson action, which contains not only the Nambu-Goldstone bosons but also the vector and axial-vector mesons. The obtained effective action consists of the nonanomalous (intrinsic parity conserving) part and the anomalous (intrinsic parity violating ) part. The nonanomalous part just coincides with the lagrangian of Bando et al. obtained on the basis of the enlarged hidden local symmetry, except that some of the parameters in their model lagrangian cannot be arbitrary in our effective lagrangian derived from the extended NJL model. A notable feature of our effective action is that not only the nonanomalous part but also the anomalous part is completely invariant under the enlarged hidden local transformation $\left(h_{L}(x), h_{R}(x)\right) \in\left[U(n)_{L} \times U(n)_{R}\right]^{(H L S)}$. Putting it in another way, there is no gauge anomaly in the enlarged hidden local symmetry. From the physical viewpoint, however, the most important symmetry of an effective action of QCD is the global chiral symmetry. If we switch off the couplings with the external gauge fields, the anomalous action that satisfies this property can easily be obtained by choosing the left-right symmetric form of regularization scheme. However, once the electroweak couplings are introduced, there arises a nontrivial problem. This is because naive use of the left-right symmetric form of regularization breaks the electromagnetic gauge invariance. To maintain the global chiral symmetry of the strong interaction together with the electromagnetic gauge invariance, we need to subtract counter terms, which depend on both the hadronic vector and axial-vector fields and the external gauge fields. This renormalization procedure enables us to obtain an effective action, which respects the global chiral symmetry at the strong interaction level as well as the electromagnetic gauge 
invariance, while keeping the full hidden local symmetry $\left[U(n)_{L} \times U(n)_{R}\right]^{(H L S)}$.

In this process of constructing an effective action consistent with the symmetries of QCD, it has become clear that the concept of the hidden local symmetry plays no positive role, which makes us to reconfirm several authors' suspicion that it may not be a physical symmetry $[4,43]$. Remember that, in our derivation of the action with enlarged hidden local symmetry, these extra gauge degrees of freedom are introduced by hand with the inclusion of two kinds of compensating fields (or "compensators"). There are several familiar examples of such compensating mechanism. A classical example is the scalar of the Stueckelberg formalism, which is used to introduce a local gauge invariance into a theory with a massive vector fields [44]. The scalar field of the chiral Schwinger model (in $1+1$ space-time dimension), which is introduced so as to cancel the chiral anomaly of the original theory [45], may also be thought of as a kind of compensator. The role of the hidden local symmetry and the associated compensating fields was discussed by de Wit and Grisaru in quite a general context [46]. Their general argument goes as follows. At the classical level, a theory with the extra gauge degrees of freedom is completely equivalent to the original theory, since the compensators can always be gauged away via the gauge transformation. Interestingly, the same is true also for theories that can be consistently quantized, since classically irrelevant gauge degrees of freedom also decouple at the quantum level, as a consequence of the Ward identities (or BRST invariance) corresponding to the classical gauge symmetry. This means that theories described with and without compensators are physically equivalent. There is one caveat in the above reasoning, however. If anomalies are present in the gauge symmetries in question, the theory becomes anomalous, i.e. it cannot be consistently quantized ; unitarity is violated, the gauge degrees of freedom no longer decouple etc. It is clear from the discussion so far 
that our effective action has no such inconsistency. Its anomalous part has been obtained as a straightforward natural of the gauged Wess-Zumino-Witten action. Owing to the function of the extra dynamical fields, i.e. the compensators, the potentially dangerous anomaly never appears. According to the expression by de Wit and Grisaru, the compensators also compensate anomaly!

At any rate, since our final effective action is completely hidden gauge invariant, the extra gauge degrees of freedom carried by the compensators can always be gauged away and decouple from all physical processes. Why ever do we consider such unphysical symmetries, then ? There are several advantages in working in a theory with extra gauge degrees of freedom. By moving freely from one gauge to another, one can get a unified view of the seemingly independent ideas. For example, we have seen that the massive Yang-Mills scheme with the (approximate) VMD type couplings with the external electroweak gauge fields can be regarded as a gauge fixed version of a lagrangian with enlarged hidden local symmetry at least formally, while we can simultaneously arrive at a clear understanding that the idea of massive Yang-Mills scheme, i.e. the full gauging of the global chiral symmetry has no theoretical foundation [47].

We also recall the fact that by introducing the extra gauge symmetries the chiral symmetries are linearly realized. Usefulness of this property may, for example, be deduced from the observation that the standard description of photon by means of a vector potential, rather than two transverse degrees of freedom, may be viewed as resulting from the introduction of a compensating mechanism used to linearlize its Lorentz transformation [46]. It is also expected to play useful roles when quantizing the theory to evaluate meson loop diagrams. Unfortunately, our effective action, though it can be consistently quantized, is not renormalizable in 
the usual power-counting sense. Recently, Gomis and Weinberg argue [48] that some gauge theories, that are not renormalizable in Dyson's sense, may nevertheless be renormalizable in the modern sense that all the divergences can be eliminated by renormalization of the infinite number of terms in the bare action. It is an interesting open question whether the concept of hidden local symmetry in effective theories of QCD may play some useful role in the context of this generalized interpretation of renormalizable theories.

\section{A Appendix}

The effective action $\Gamma_{L R}^{(a)}$ derived in sect.2 can trivially be generalized to arbitrary even-dimensional space-time case ( $n=D / 2$ with $D$ being the space-time dimension) as

$$
\begin{gathered}
\Gamma_{L R}^{(a)}=c_{n} \int_{B^{2 n+1}}\left[\omega_{2 n+1}^{0}\left(0, \xi_{R}^{\dagger} d \xi_{R}\right)-\omega_{2 n+1}^{0}\left(0, \xi_{L}^{\dagger} d \xi_{L}\right)-\omega_{2 n+1}^{0}\left(0, \xi_{M} d \xi_{M}^{\dagger}\right)\right] \\
+c_{n} \int_{S^{2 n}}\left[\rho_{2 n}\left(0, R, \xi_{R}^{\dagger} d \xi_{R}\right)+\rho_{2 n}\left(0, \xi_{L}^{\dagger} d \xi_{L}, L\right)\right. \\
\left.\quad+\rho_{2 n}\left(0, \xi_{M} d \xi_{M}^{\dagger}, \tilde{L}\right)-\rho_{2 n}(0, \hat{L}, \hat{R})\right]
\end{gathered}
$$

with $c_{n}=(-i)^{n+1} N_{c} /(2 \pi)^{n}(n+1)$ !. The responses of the above action under an arbitrary gauge variations can easily be evaluated by using the method described in [39]. First, we show the response of each term of (A.1) under the $\left[U(n)_{L} \times U(n)_{R}\right]^{(H L S)}$ transformation. Under the infinitesimal left variation $\left(h_{L}=e^{-\epsilon_{L}} \simeq 1-\epsilon_{L}, h_{R}=1\right)$, we find that

$$
\begin{aligned}
\delta_{L}^{(H L S)} \omega_{2 n+1}^{0}\left(0, \xi_{R}^{\dagger} d \xi_{R}\right) & =0 \\
\delta_{L}^{(H L S)} \omega_{2 n+1}^{0}\left(0, \xi_{L}^{\dagger} d \xi_{L}\right) & =(-1)^{n+1} \frac{(n+1)(n !)^{2}}{(2 n) !} d \operatorname{tr} \epsilon_{L}\left(\xi_{L} d \xi_{L}^{\dagger}\right)^{2 n} \\
\delta_{L}^{(H L S)} \omega_{2 n+1}^{0}\left(0, \xi_{M} d \xi_{M}^{\dagger}\right) & =(-1)^{n} \frac{(n+1)(n !)^{2}}{(2 n) !} d \operatorname{tr} \epsilon_{L}\left(\xi_{M} d \xi_{M}^{\dagger}\right)^{2 n}
\end{aligned}
$$




$$
\begin{aligned}
\delta_{L}^{(H L S)} \rho_{2 n}\left(0, R, \xi_{R}^{\dagger} d \xi_{R}\right) & =0 \\
\delta^{(H L S)} \rho_{2 n}\left(0, \xi_{L}^{\dagger} d \xi_{L}, L\right) & =(-1)^{n} \frac{(n+1)(n !)^{2}}{(2 n) !} \operatorname{tr} \epsilon_{L}\left(\xi_{L} d \xi_{L}^{\dagger}\right)^{2 n}-\bar{\Delta}\left(\epsilon_{L}, \tilde{L}\right), \\
\delta_{L}^{(H L S)} \rho_{2 n}\left(0, \xi_{M} d \xi_{M}^{\dagger}, \tilde{L}\right) & =(-1)^{n} \frac{(n+1)(n !)^{2}}{(2 n) !} \operatorname{tr} \epsilon_{L}\left(\xi_{M} d \xi_{M}^{\dagger}\right)^{2 n}+\bar{\Delta}\left(\epsilon_{L}, \tilde{L}\right), \\
\delta_{L}^{(H L S)} & \rho_{2 n}(0, \hat{L}, \hat{R})=0 .
\end{aligned}
$$

Here we have defined the quantity :

$$
\bar{\Delta}(\theta, A)=(n+1) \sum_{p=0}^{n-1} \int_{0}^{1} d s(1-s) \operatorname{tr}\left[d \theta\left(s d A+s^{2} A^{2}\right)^{p} A\left(s d A+s^{2} A^{2}\right)^{n-p-1}\right] .
$$

For $n=D / 2=2$, this reduces to

$$
\bar{\Delta}(\theta, A)=\operatorname{tr} d \theta \frac{1}{2}\left(A d A+d A A+A^{3}\right) .
$$

On the other hand, under the infinitesimal right variation $\left(h_{L}=1, h_{R}=e^{-\epsilon_{R}} \simeq 1-\epsilon_{R}\right)$, we have

$$
\begin{aligned}
\delta_{R}^{(H L S)} \omega_{2 n+1}^{0}\left(0, \xi_{R}^{\dagger} d \xi_{R}\right) & =(-1)^{n+1} \frac{(n+1)(n !)^{2}}{(2 n) !} d \operatorname{tr} \epsilon_{R}\left(\xi_{R} d \xi_{R}^{\dagger}\right)^{2 n} \\
\delta_{R}^{(H L S)} \omega_{2 n+1}^{0}\left(0, \xi_{L}^{\dagger} d \xi_{L}\right) & =0 \\
\delta_{R}^{(H L S)} \omega_{2 n+1}^{0}\left(0, \xi_{M} d \xi_{M}^{\dagger}\right) & =(-1)^{n+1} \frac{(n+1)(n !)^{2}}{(2 n) !} d \operatorname{tr} \epsilon_{R}\left(\xi_{M}^{\dagger} d \xi_{M}\right)^{2 n} \\
\delta_{R}^{(H L S)} \rho_{2 n}\left(0, R, \xi_{R}^{\dagger} d \xi_{R}\right) & =(-1)^{n} \frac{(n+1)(n !)^{2}}{(2 n) !} \operatorname{tr} \epsilon_{R}\left(\xi_{R} d \xi_{R}^{\dagger}\right)^{2 n}+\bar{\Delta}\left(\epsilon_{R}, \tilde{R}\right) \\
\delta_{R}^{(H L S)} \rho_{2 n}\left(0, \xi_{L}^{\dagger} d \xi_{L}, L\right) & =0 \\
\delta_{R}^{(H L S)} \rho_{2 n}\left(0, \xi_{M} d \xi_{M}^{\dagger}, \tilde{L}\right) & =(-1)^{n+1} \frac{(n+1)(n !)^{2}}{(2 n) !} \operatorname{tr} \epsilon_{R}\left(\xi_{M}^{\dagger} d \xi_{M}\right)^{2 n}-\bar{\Delta}\left(\epsilon_{R}, \hat{L}\right), \\
\delta_{R}^{(H L S)} \rho_{2 n}(0, \hat{L}, \hat{R}) & =\bar{\Delta}\left(\epsilon_{R}, \tilde{R}\right)-\bar{\Delta}\left(\epsilon_{R}, \hat{L}\right) .
\end{aligned}
$$

One can easily convince that, for either of the right or left hidden gauge variation, the responses of the individual terms cancel out to be zero, thereby leading to the result :

$$
\delta_{L}^{(H L S)} \Gamma_{L R}^{(a)}=\delta_{R}^{(H L S)} \Gamma_{L R}^{(a)}=0,
$$


which denotes that $\Gamma^{(a)}$ is completely invariant under the enlarged hidden local symmetry.

We can similarly evaluate the response of each term of $\Gamma^{(a)}$ under the external gauge variations belonging to $\left[U(n)_{L} \times U(n)_{R}\right]^{(e x t)}$. Under the infinitesimal left variation $\left(g_{L}=\right.$ $e^{-\theta_{L}} \simeq 1-\theta_{L}, g_{R}=1$ ), we find that

$$
\begin{aligned}
\delta_{L}^{(e x t)} \omega_{2 n+1}^{0}\left(0, \xi_{R}^{\dagger} d \xi_{R}\right) & =0, \\
\delta_{L}^{(e x t)} \omega_{2 n+1}^{0}\left(0, \xi_{L}^{\dagger} d \xi_{L}\right) & =(-1)^{n} \frac{(n+1)(n !)^{2}}{(2 n) !} d \operatorname{tr} \theta_{L}\left(\xi_{L}^{\dagger} d \xi_{L}\right)^{2 n}, \\
\delta_{L}^{(e x t)} \omega_{2 n+1}^{0}\left(0, \xi_{M} d \xi_{M}^{\dagger}\right) & =0, \\
\delta_{L}^{(e x t)} \rho_{2 n}\left(0, R, \xi_{R}^{\dagger} d \xi_{R}\right) & =0, \\
\delta^{(e x t)} \rho_{2 n}\left(0, \xi_{L}^{\dagger} d \xi_{L}, L\right) & =(-1)^{n} \frac{(n+1)(n !)^{2}}{(2 n) !} \operatorname{tr} \theta_{L}\left(\xi_{L}^{\dagger} d \xi_{L}\right)^{2 n}+\bar{\Delta}\left(\theta_{L}, L\right), \\
\delta_{L}^{(e x t)} \rho_{2 n}\left(0, \xi_{M} d \xi_{M}^{\dagger}, \tilde{L}\right) & =0, \\
\delta_{L}^{(e x t)} \rho_{2 n}(0, \hat{L}, \hat{R}) & =0 .
\end{aligned}
$$

On the other hand, the infinitesimal right variation $\left(g_{L}=1, g_{R}=e^{-\theta_{R}} \simeq 1-\theta_{R}\right)$ gives

$$
\begin{aligned}
\delta_{R}^{(e x t)} \omega_{2 n+1}^{0}\left(0, \xi_{R}^{\dagger} d \xi_{R}\right) & =(-1)^{n} \frac{(n+1)(n !)^{2}}{(2 n) !} d \operatorname{tr} \theta_{R}\left(\xi_{R}^{\dagger} d \xi_{R}\right)^{2 n}, \\
\delta_{R}^{(e x t)} \omega_{2 n+1}^{0}\left(0, \xi_{L}^{\dagger} d \xi_{L}\right) & =0 \\
\delta_{R}^{(e x t)} \omega_{2 n+1}^{0}\left(0, \xi_{M} d \xi_{M}^{\dagger}\right) & =0 \\
\delta_{R}^{(e x t)} \rho_{2 n}\left(0, R, \xi_{R}^{\dagger} d \xi_{R}\right) & =(-1)^{n+1} \frac{(n+1)(n !)^{2}}{(2 n) !} \operatorname{tr} \theta_{R}\left(\xi_{R}^{\dagger} d \xi_{R}\right)^{2 n}-\bar{\Delta}\left(\theta_{R}, R\right), \\
\delta_{R}^{(e x t)} \rho_{2 n}\left(0, \xi_{L}^{\dagger} d \xi_{L}, L\right) & =0 \\
\delta_{R}^{(e x t)} \rho_{2 n}\left(0, \xi_{M} d \xi_{M}^{\dagger}, \tilde{L}\right) & =0 \\
\delta_{R}^{(e x t)} \rho_{2 n}(0, \hat{L}, \hat{R}) & =0 .
\end{aligned}
$$

Summing up all the contributions, we are led to the expected result, i.e. the left-right sym- 
metric form of anomaly given as,

$$
\begin{aligned}
\delta_{L}^{(e x t)} \Gamma_{L R}^{(a)} & =c_{n} \int_{S^{4}} \bar{\Delta}\left(\theta_{L}, L\right) \\
\delta_{R}^{(e x t)} \Gamma_{L R}^{(a)} & =-c_{n} \int_{S^{4}} \bar{\Delta}\left(\theta_{R}, R\right) .
\end{aligned}
$$

Note that $\bar{\Delta}(\theta, A)$ is proportional to $d \theta$ (see (A.9) or (A.10)). It is then obvious that, specializing to the global chiral transformation, the right hand sides of (A.33) and (A.34) both vanish. 


\section{References}

[1] S. Gasiorowicz and D. A. Geffen, Rev. Mod. Phys. 41, 531 (1969).

[2] J. J. Sakurai, Currents and Mesons (University of Chicago Press, Chicago, 1969).

[3] J. F. Donoghue, E. Golowich and B. R. Holstein, Dynamics of the Standard Model, (Cambridge University Press, Cambridge, 1992).

[4] M. C. Birse, "Effective chiral Lagrangians for spin-1 mesons", hep-ph-9603251 (1996).

[5] U.-G. Meissner, Phys. Rep. 161, 213 (1988).

[6] M. Bando, T. Kugo and K. Yamawaki, Phys. Rep. 164, 217 (1988).

[7] M. Bando, T. Kugo, S. Uehara, K. Yamawaki and T. Yanagida, Phys. Rev. Lett. 54, 1215 (1985).

[8] M. Bando, T. Kugo and K. Yamawaki, Prog. Theor. Phys. 73, 1541 (1985).

[9] M. Bando, T. Fujiwara and K. Yamawaki, Prog. Theor. Phys. 79, 1140 (1988).

[10] S. Weiberg, Phys. Rev. 166, 1568 (1968).

[11] S. Coleman, J. Wess and B. Zumino, Phys. Rev. 177, 2239 (1969) ;

C. G. Callan, S. Coleman, J. Wess and B. Zumino, Phys. Rev. 177, 2247 (1969).

[12] G. Ecker, H. Leutwyler, J. Gasser, A. Pich and E. de Rafael, Phys. Lett. B223, 425 (1989). 
[13] E. Cremmer and B. Julia, Phys. Lett. B80, 48 (1978) ;

Nucl. Phys. B159, 141 (1979).

[14] S. J. Gates Jr., M. T. Grisaru, M. Roček and W. Siegel, Superspace (Benjamin / Cummings, New York, 1983) Chap.3.

[15] A. D'Adda, P. Di Vecchia and M. Lüscher, Nucl. Phys. B146, 63 (1978) ; Nucl. Phys. B152, 125 (1979).

[16] D. Ebert and M. K. Volkov, Z. Phys. C16, 205 (1983).

[17] M. K. Volkov, Ann. Phys. (NY) 157, 282 (1984).

[18] D. Ebert and H. Reinhardt, Nucl. Phys. B271, 188 (1986).

[19] A. Dhar, R. Shankar and S. Wadia, Phys. Rev. D31, 3256 (1985).

[20] R. Ball, in Skyrmion and Anomalies, edited by M. Jezabek and M. Praszałowicz (World Scientific, Singapore, 1987).

[21] M. Wakamatsu and W. Weise, Z. Phys. A331, 173 (1988).

[22] M. Wakamatsu, Ann. Phys. 193, 287 (1989).

[23] E. Ruiz Arriola and L. L. Salcedo, Phys. Lett. B316, 148 (1993).

[24] J. Bijnens, Ch. Bruno and E. de Rafael, Nucl. Phys. B390, 501 (1993).

[25] T. Eguchi and H. Sugawara, Phys. Rev. D10, 4275 (1974) ; T. Eguchi, Phys. Rev. D14, 2755 (1976).

[26] K. Kikkawa, Prog. Theor. Phys. 56, 947 (1976). 
[27] T. Kugo, Prog. Theor. Phys. 55, 2032 (1976).

[28] H. Gomm, Ö. Kaymakcalan and J. Schechter, Phys. Rev. D30, 2345 (1984).

[29] Y. Brihake, N. K. Pak and P. Rossi, Nucl. Phys. B254, 71 (1985).

[30] Ö. Kaymakcalan and J. Schechter, Phys. Rev. D31, 1109 (1985).

[31] J. Schechter, Phys. Rev. D34, 868 (1986).

[32] J. Bijnens and J. Prades, Phys. Lett. B320, 130 (1994).

[33] E. Ruiz Arriola and L. L. Salcedo, Nucl Phys. A590, 703 (1995).

[34] G. 't Hooft, Phys. Rep. 142, 357 (1986).

[35] K. Fujikawa, Phys. Rev. D21, 2848 (1980) ; Phys. Rev. D23, 2262 (1981).

[36] B. Zumino, Y. S. Wu and A. Zee, Nucl. Phys. B239, 477 (1984).

[37] B. Zumino, in Relativity, Group and Topology, edited by B. S. de Witt and S. Stora (North Holland, Amsterdam) p.1291.

[38] J. L. Mañes, Nucl. Phys. B250, 369 (1985).

[39] J. L. Petersen, Acta. Phys. Pol. B16, 271 (1985).

[40] W. A. Bardeen, Phys. Rev. 184, 1848 (1969).

[41] J. Wess and B. Zumino, Phys. Lett. 37B, 95 (1971).

[42] E. Witten, Nucl. Phys. B223, 422 (1983).

[43] H. Georgi, Phys. Rev. Lett. 63, 1917 (1989) ; Nucl. Phys. B331, 311 (1990). 
[44] E. C. G. Stueckelberg, Helv. Phys. Acta. 11, 225 (1938).

[45] L. D. Faddeev and S. L. Shatashvili, Phys. Lett. B167, 225 (1986) ;

L. D. Faddeev, Phys. Lett. B145, 81 (1984).

[46] B. de Wit and M. T. Grisaru, in Quantum Field Theory and Quantum Statistics, Vol. 2 : Models of Field Theory, edited by I. A. Batalin, C. J.!Isham and G. A. Vilkovsky (Adam Hilgar, Bristol, 1987) p.411.

[47] K. Yamawaki, Phys. Rev. D35, 412 (1987).

[48] J. Gomis and S. Weinberg, "Are Nonrenormalizable Gauge Theories Renormalizable ?", hep-th-9510087 (1995).

\section{Figure caption}

Fig. 1(a). The integral path in the field space for obtaining the anomalous action with the symmetry $G_{\text {global }} \times G_{\text {local }}$ with $G_{\text {global }}=\left[U(n)_{L} \times U(n)_{R}\right]^{(e x t)}$ and $G_{\text {local }}=\left[U(n)_{L} \times U(n)_{R}\right]^{(H L S)}$.

Fig. 1(b). The decomposition of the integral path of fig.1(a) into two parts, which respectively correspond to the contributions from the Jacobians $J_{1}$ and $J_{2}$ in $(2.65)$. 


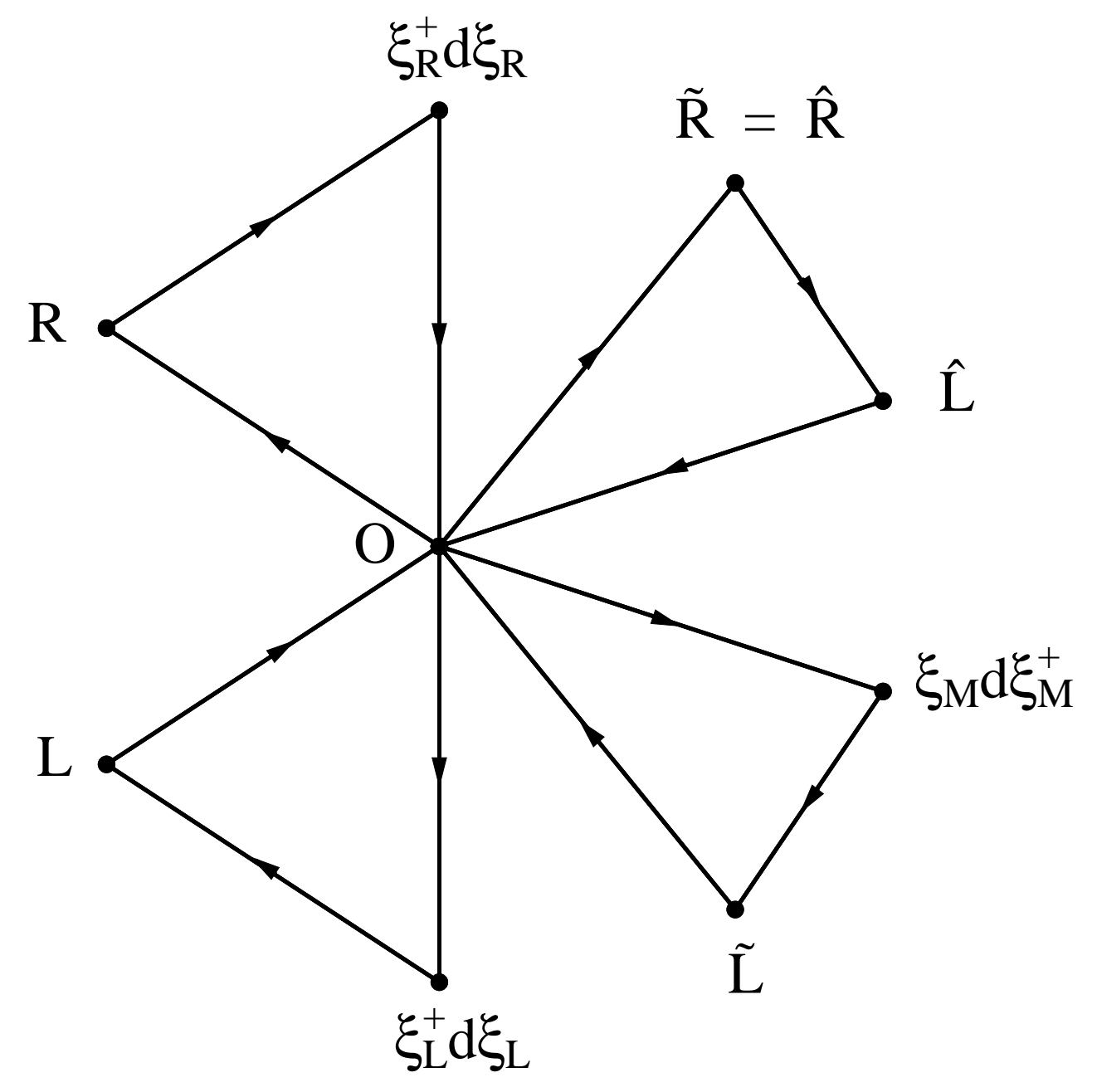

Fig.1(a) 


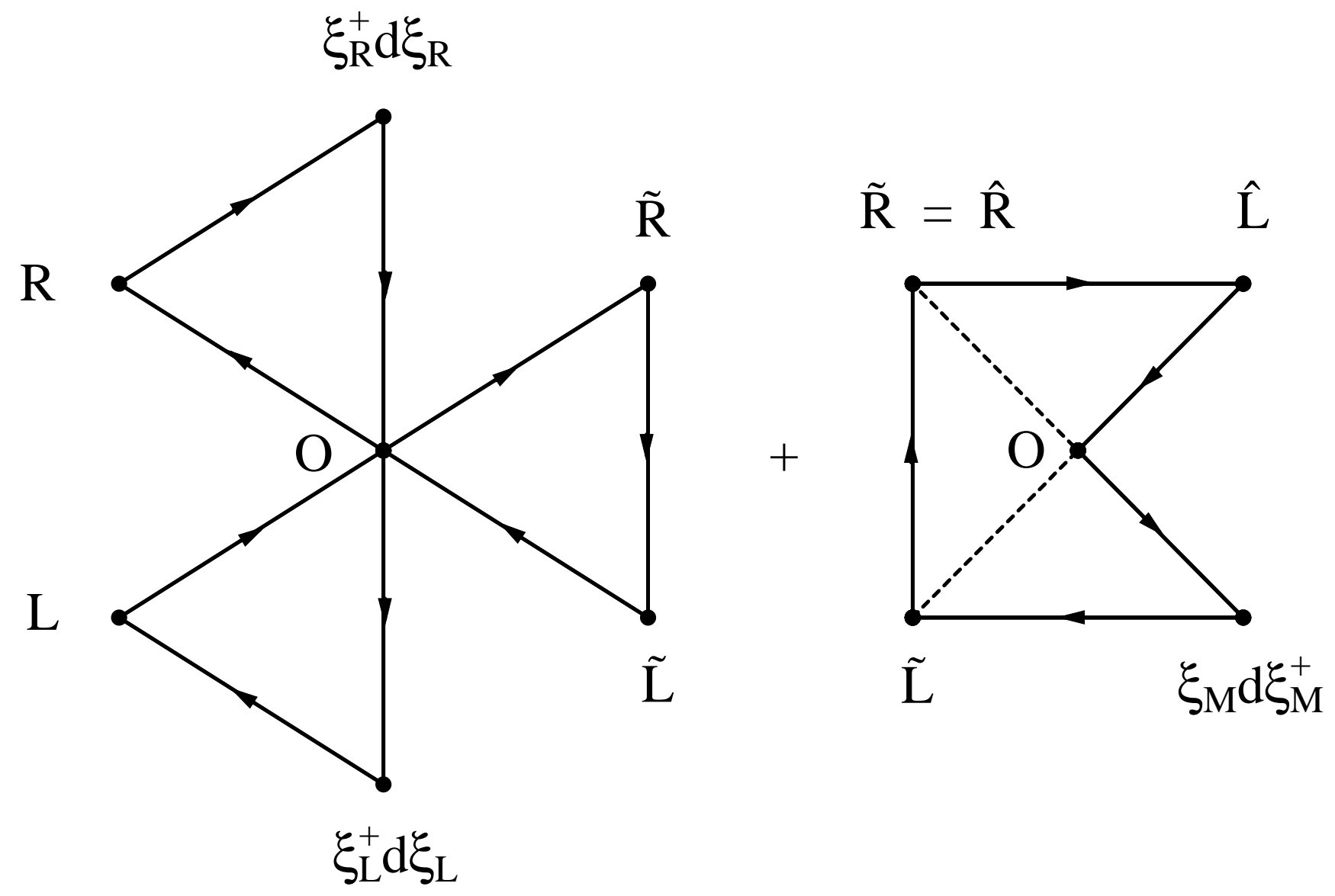

Fig. 1(b) 